\title{
The Large APEX BOlometer CAmera LABOCA
}

\author{
G. Siringo ${ }^{1}$, E. Kreysa ${ }^{1}$, A. Kovács ${ }^{1}$, F. Schuller ${ }^{1}$, A. Weiß ${ }^{1}$, W. Esch ${ }^{1}$, H.-P. Gemünd ${ }^{1}$, N. Jethava ${ }^{2}$, \\ G. Lundershausen ${ }^{1}$, A. Colin ${ }^{3}$, R. Güsten ${ }^{1}$, K. M. Menten ${ }^{1}$, A. Beelen ${ }^{4}$, F. Bertoldi ${ }^{5}$, J. W. Beeman ${ }^{6}$, and E. E. Haller ${ }^{6}$ \\ 1 Max-Planck-Institut für Radioastronomie, Auf dem Hügel 69, 53121 Bonn, Germany \\ e-mail: gsiringo@mpifr-bonn.mpg.de \\ 2 National Institute of Standards and Technology, Boulder, CO 80305, USA \\ 3 Instituto de Fisica de Cantabria (CSIC-UC), Avda. Los Castros, 39005 Santander, Spain \\ 4 Institut d'Astrophysique Spatiale, Bât. 121, Université Paris-Sud, 91405 Orsay Cedex, France \\ 5 Argelander-Institut für Astronomie, University of Bonn, Auf dem Hügel 71, 53121 Bonn, Germany \\ ${ }^{6}$ Lawrence Berkeley National Laboratory, Berkeley, CA 94720, USA
}

Received 1 December 2008 / Accepted 21 January 2009

\begin{abstract}
The Large APEX BOlometer CAmera, LABOCA, has been commissioned for operation as a new facility instrument at the Atacama Pathfinder Experiment $12 \mathrm{~m}$ submillimeter telescope. This new 295-bolometer total power camera, operating in the $870 \mu \mathrm{m}$ atmospheric window, combined with the high efficiency of APEX and the excellent atmospheric transmission at the site, offers unprecedented capability in mapping submillimeter continuum emission for a wide range of astronomical purposes.
\end{abstract}

Key words. instrumentation: detectors - instrumentation: photometers - submillimeter - methods: observational

\section{Introduction}

\subsection{Astronomical motivation}

Millimeter and submillimeter wavelength continuum emission is a powerful probe of the warm and cool dust in the Universe. For temperatures below $\sim 40 \mathrm{~K}$, the peak of the thermal continuum emission is at wavelengths longer than $100 \mu \mathrm{m}$ (or at frequencies lower than $3 \mathrm{THz}$ ), i.e. in the far-infrared and (sub)millimeter ${ }^{1}$ range. A number of atmospheric windows between $200 \mathrm{GHz}$ and $1 \mathrm{THz}$ make ground-based observations possible over a large part of this range from high-altitude, dry sites.

Specifically, thermal dust emission is well described by a gray body spectrum, with the measured flux density $S_{v}$ at frequency $v$ expressed as:

$S_{v}=\Omega_{\mathrm{S}^{\prime}}\left(1-\mathrm{e}^{-\tau}\right) \frac{2 h}{c^{2}} \frac{v^{3}}{\mathrm{e}^{h v / k T}-1}$

where $h$ and $k$ are Planck's and Boltzmann's constants respectively, $c$ is the speed of light, $\Omega_{S^{\prime}}$ is the apparent source solid angle (the size of the physical source convolved with the telescope beam) and $\tau$ is the optical depth, which varies with frequency. In the (sub)millimeter range, the emission is almost always optically thin with $\tau \propto N \nu^{\beta}$, where $\beta$ is in the range 12 typically (see, e.g., the Appendix of Mezger et al. 1990; or Beuther et al. 2002, for a more thorough discussion). Here, $N$ is the number of dust particles (or number of nucleons assuming a given dust-to-gas relation) in the telescope beam. Accordingly, the (sub)millimeter flux can be converted into dust/gas masses,

\footnotetext{
${ }^{1}$ Hereafter we will use the term (sub)millimeter when referring, in general, to the millimeter and submillimeter wavelengths range; we will use submillimeter when strictly referring to wavelengths from one millimeter down to the far-infrared.
}

when the temperature $T$ is assumed or constrained via additional far-infrared measurements.

Such mass determination is one of the core issues of (sub)millimeter photometry. We would like to illustrate its paramount importance with a few examples: (sub)millimeter wavelengths mapping of low-mass star-forming regions in molecular clouds have determined the dense core mass spectrum, (in nearby regions) down to sub-stellar masses, and investigated its relationship to the Initial Mass Function (Motte et al. 1998). These studies can be extended to high-mass starforming regions (e.g. Motte et al. 2007; Johnstone et al. 2006). Because of the larger distances to rarer, high-mass embedded objects, even relatively shallow surveys are capable of detecting pre-stellar cluster clumps with a few hundreds of solar masses of material at distances as far as the Galactic center. Masses and observed sizes yield radial density distribution profiles for protostellar cores that can be compared with theoretical models (Beuther et al. 2002).

(Sub)millimeter continuum emission is also a remarkable tool for the study of the distant Universe. The thermal dust emission in active galaxies is typically fueled by short-lived, high-mass stars, therefore the far-infrared luminosity provides a snapshot of the current level of star-formation activity. Deep (sub)millimeter observations in the Hubble Deep Field, with the Submillimeter Common User Bolometer Array (SCUBA, Holland et al. 1999) on the James Clerk Maxwell Telescope, attracted considerable attention with the detection of a few sources without optical or near-infrared counterpart. Sensitive measurements found dust in high redshift sources and even in some of the farthest known objects in the Universe (see, e.g., Carilli et al. 2001; Bertoldi et al. 2003; Wang et al. 2008), revealing starformation rates (SFRs) that are hundreds of times higher than in the Milky Way today. These detections are possible because 
of the so-called negative K-correction first discussed by Blain \& Longair (1993): the warm dust in galaxies is typically characterized by temperatures around $30-60 \mathrm{~K}$. Its thermal emission dominates the spectral energy distribution (SED) of luminous and ultra-luminous infrared galaxies (LIRGs and ULIRGs), which have maxima between 3 and $6 \mathrm{THz}$ as a result. Because of the expansion of the Universe, the peak of the emission shifts toward the lower frequencies with increasing cosmological distance, thus counteracting the dimming and benefitting detection at (sub)millimeter wavelength. Consequently, flux-limited surveys near millimeter wavelengths yield flat or nearly flat luminosity selection over much of the volume of the Universe (Blain et al. 2002). As such, (sub)millimeter wavelengths allow unbiased studies of the luminosity evolution and, therefore, of the star-formation history of galaxies over cosmological time-scales.

\subsection{Bolometer arrays for (sub)millimeter astronomy}

The (sub)millimeter dust emission is unfortunately intrinsically weak and the wish to measure it pushed the development of detectors with the best possible sensitivity, namely bolometers (Low 1961; Mather 1984). Moreover, the desirability of mapping large areas of the sky, motivated the development of detector arrays. Consequently, the last 10 years have seen an increasing effort in the development of bolometer arrays. In such instruments, a number of composite bolometers work side by side in the focal plane, offering simultaneous multi-beam coverage. Since the arrival of the first arrays, developed in the early ' 90 s and consisting of just 7 elements, we are witnessing a rapid maturing of technology, reaching hundreds to a few thousand elements today, and the prospect of even larger bolometer arrays in the future. The Large APEX BOlometer CAmera, LABOCA, described in this article, is a new bolometric receiver array for the Atacama Pathfinder Experiment $12 \mathrm{~m}$ telescope, $\mathrm{APEX}^{2}$. It is the most ambitious camera in a long line of developments of the MPIfR bolometer group, which has delivered instruments of increasing complexity to the IRAM 30 m telescope (Kreysa 1990): single beam receivers were supplanted by a 7-element system (Kreysa et al. 1999), which eventually gave way to the MAx-Planck Millimeter BOlometer (MAMBO) array, whose initial 37 beams have grown to 117 in the latest incarnation (Kreysa et al. 2002). The group also built the 37-element $1.2 \mathrm{~mm}$ SEST Imaging Bolometer Array (SIMBA) for the Swedish/ESO Submillimeter telescope (Nyman et al. 2001) and the 19-element $870 \mu \mathrm{m}$ for the $10 \mathrm{~m}$ Heinrich-Hertz Telescope (a.k.a. SMTO, Martin \& Baars 1990).

The main obstacle to observations at these wavelengths is posed by Earth's atmosphere, which is seen as a bright emitting screen by a continuum total power detector, as LABOCA's bolometers are. This is largely due to the emission of the water vapor present in the atmosphere with only small contributions from other components, like ozone. Besides, the atmosphere is a turbulent thermodynamic system and the amount of water vapor along the line of sight can change quickly, giving rise to instabilities of emission and transmission, called sky noise. Observations from ground based telescopes have to go through that screen, therefore requiring techniques to minimize those effects.

The technique most widely used is to operate a switching device, usually a chopping secondary mirror (hereafter called

\footnotetext{
${ }^{2}$ APEX is a collaborative effort between the Max-Planck-Institut für Radioastronomie of Bonn (MPIfR, 50\%), the European Southern Observatory (ESO, 27\%) and the Onsala Space Observatory (OSO, 23\%).
}

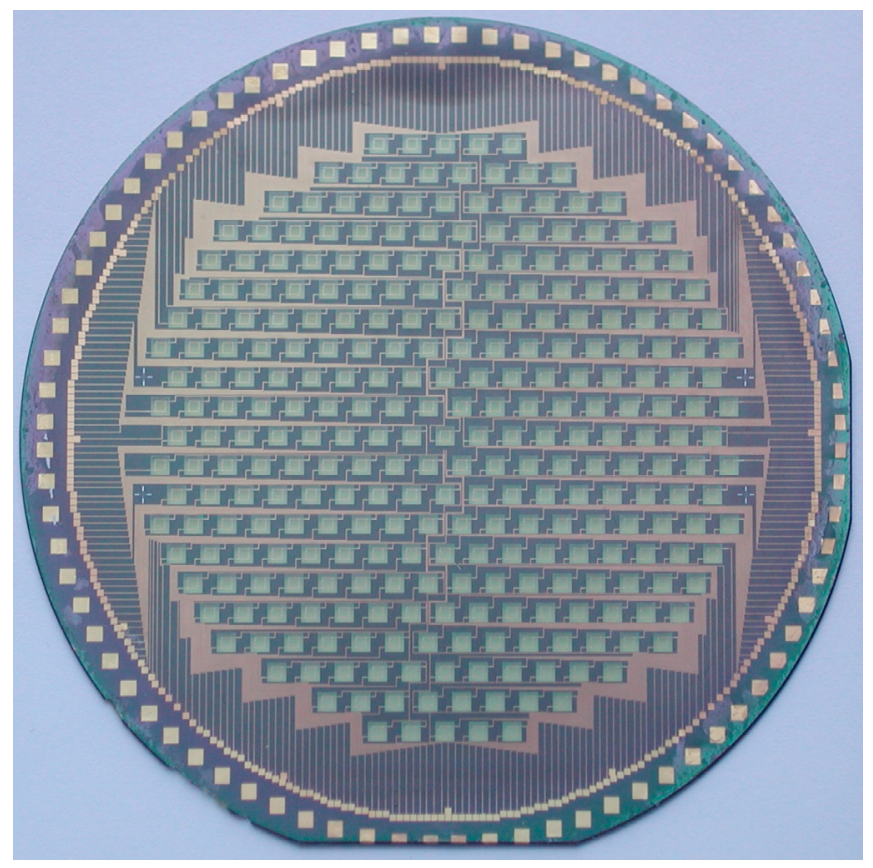

Fig. 1. Wiring side of a naked LABOCA array. Each light-green square is a bolometer.

wobbler) to observe alternatively the source and a blank sky area close to it, at a frequency higher than the variability scale of the sky noise. This method, originally introduced for observations with single pixel detectors, is today also used with arrays of bolometers. Although it can be very efficient to reduce the atmospheric disturbances during observations, it presents some disadvantages: among others, the wobbler is usually slow (1 or $2 \mathrm{~Hz}$ ) posing a strong limitation to the possible scanning speed.

LABOCA has been specifically designed to work without a wobbler and using a different technique, which works particularly well when using an array of detectors, to remove the atmospheric contribution. This technique, called fast scanning (Reichertz et al. 2001), is based on the idea that, when observing with an array, the bolometers composing the array look simultaneously at different points in the sky, therefore chopping is no more needed. A modulation of the signal, still required to identify the astronomical source through the atmospheric emission, is produced by scanning with the telescope across the source. The atmospheric contribution (as well as part of the instrumental noise) will be strongly correlated in all bolometers and a post-detection analysis of the correlation across the array will make it possible to extract the signals of astronomical interest from the atmospheric foregrounds. Moreover, the post-detection bandwidth depends on the scanning speed, therefore relatively high scanning speeds are ideal (see also Kovács 2008).

The APEX telescope (Güsten et al. 2006), as the name implies, serves as a pathfinder for the future large-scale (sub)millimeter wavelength and (far)infrared missions, namely the Atacama Large Millimeter Array (ALMA), the Herschel Space Observatory and the Stratospheric Observatory for Infrared Astronomy (SOFIA). Its pathfinder character is on the one hand defined by exploring wavelength windows that have been poorly studied before, with acceptable atmospheric transmission at the $5100 \mathrm{~m}$ altitude site. On the other hand, and more importantly, it can perform large area mapping to identify interesting sources for ALMA follow-up studies at higher angular resolution. Moreover, APEX produces images of both 


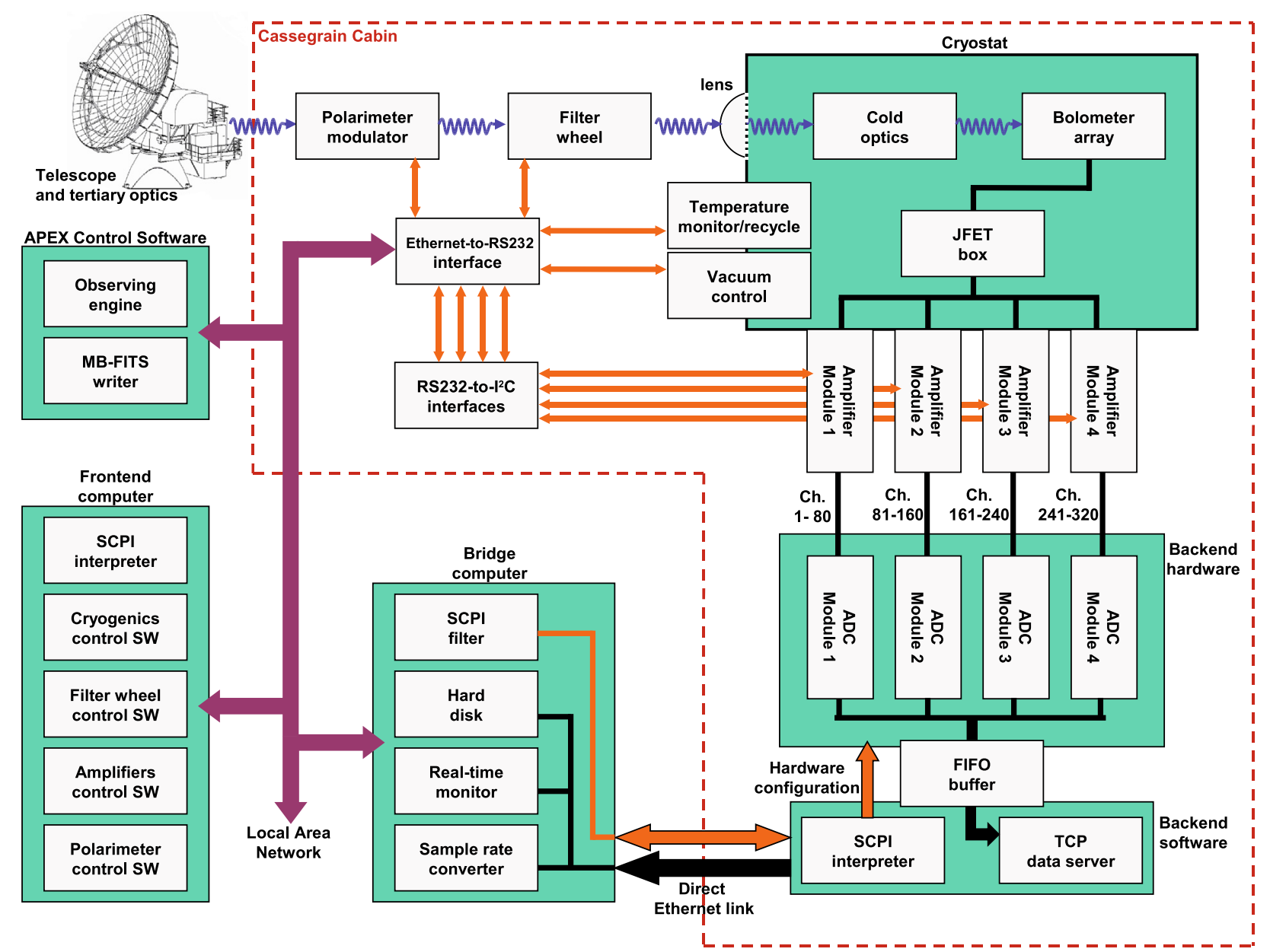

Fig. 2. Scheme of the infrastructure of LABOCA. The instrument is located in the Cassegrain cabin (dashed red line) of APEX, remote operation is a requirement and all the communication goes through the local area network (magenta arrows) and a direct Ethernet link between backend and bridge computers. The black lines show the flow of the bolometer signals, the orange ones show the configuration and monitoring communication.

continuum (with LABOCA) and line emission with angular resolutions that neither Herschel's nor SOFIA's smaller telescopes (with diameters of 3.5 and $2.5 \mathrm{~m}$ respectively) can match. This provides a critical advantage to APEX for imaging dust and line emission at high frequencies.

\subsection{Instrument overview}

LABOCA is an array of bolometers, operated in total-power mode, specifically designed for fast mapping of large areas of sky at moderate resolution and with high sensitivity and was commissioned in May 2007 as facility instrument on APEX. It is a very complex system, composed of parts that originate in a variety of fields of technology, in particular optics, high vacuum, low temperature cryogenics, digital electronics, computer hardware and software, and others. A general view of the infrastructure is shown in the block diagram of Fig. 2. The heart of LABOCA is its detector array made of 295 semiconducting composite bolometers (see Figs. 1, 6). A description of the detector array design and manufacture is provided in Sect. 5.

The bolometer array is mounted in a cryostat, which uses liquid nitrogen and liquid helium on a closed cycle double-stage sorption cooler to reach an operation temperature of $\sim 285 \mathrm{mK}$. The cryogenic system is discussed in Sect. 3.

A set of cold filters, mounted on the liquid nitrogen and liquid helium shields, define the spectral passband, centered at a wavelength of $870 \mu \mathrm{m}$ and about $150 \mu \mathrm{m}$ wide (see Fig. 5). A monolithic array of conical horn antennas, placed in front of the bolometer wafer, concentrates the radiation onto the individual bolometers. The filters and the horn array are presented in Sect. 4.

The cryostat is located in the Cassegrain cabin of the APEX telescope (see Fig. 4) and the optical coupling to the telescope is provided by an optical system made of a series of metal mirrors and a lens which forms the cryostat window. The complex optics layout, manufacture and installation at the telescope is described in Sect. 2.

The bolometer signals are routed through low noise, unity gain Junction Field Effect Transistor (JFET) amplifiers and to the outside of the cryostat along flexible flat cables. Upon exiting the cryostat, the signals pass to room temperature low noise amplifiers and electronics also providing the AC current for biasing the bolometers and performing real time demodulation of the signals. The signals are then digitized over 16 bits by 4 data acquisition boards providing 80 analog inputs each, mounted in the backend computer. The backend software provides an interface to the telescope's control software, used to set up the hardware, and a data server for the data output. The acquired data are then digitally filtered and downsampled to a lower rate in real time by the bridge computer and finally stored in MB-FITS format (Muders et al. 2006) by the FITS writer embedded in the telescope's control software. Another computer, the frontend 

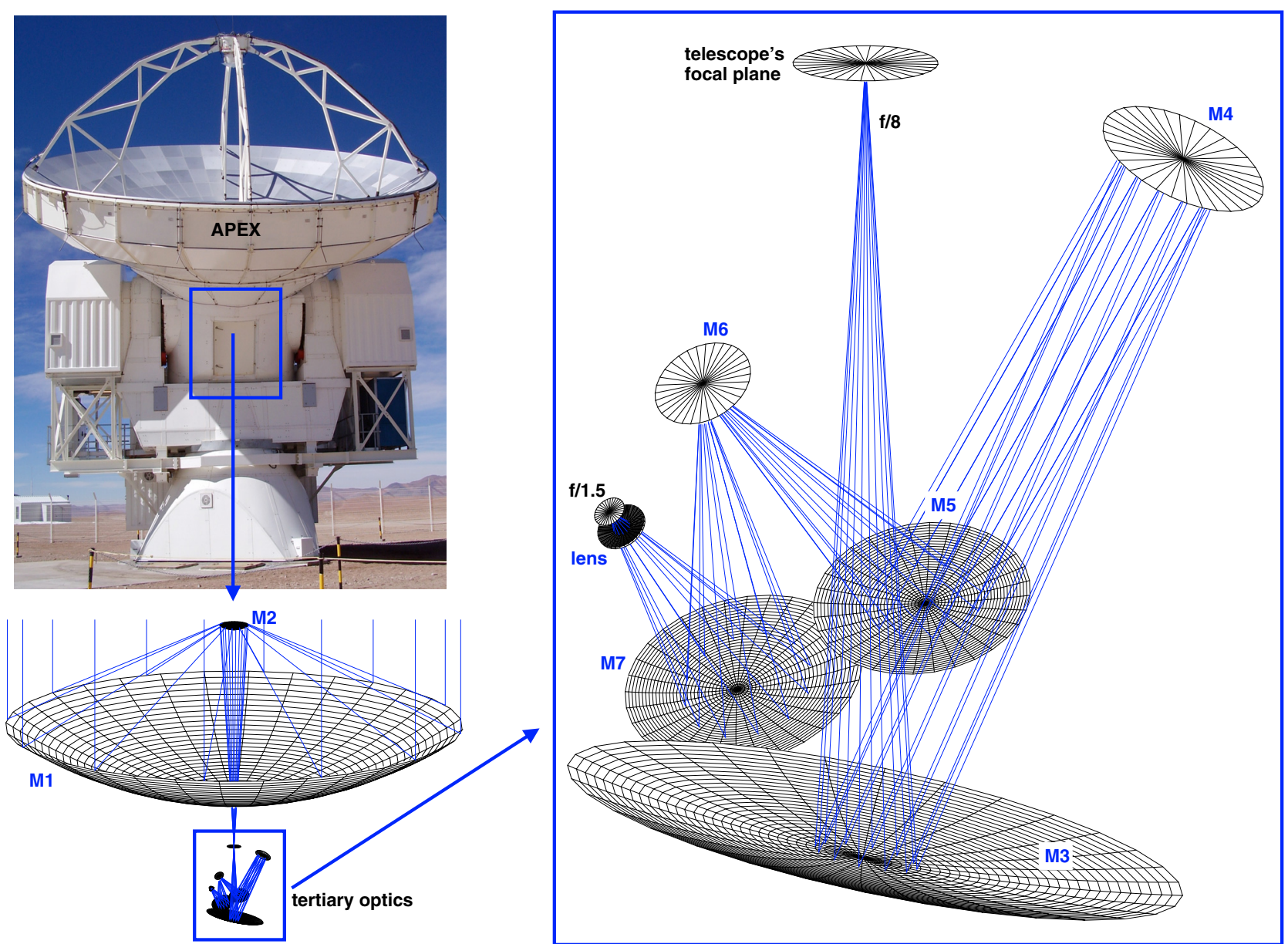

Fig. 3. Overview of the optics. On the left, the APEX telescope. On the right, a zoom on the tertiary optics installed in the Cassegrain cabin. See also Fig. 4.

computer, is devoted to monitor and control most of the electronics embedded into the receiver (e.g. monitoring of all the temperature stages, controlling of the sorption cooler, calibration unit) and provides an interface to the APEX control software, allowing remote operation of the system. Discussions of the cold and warm readout electronics, plus the signal processing, are found in Sects. 6 and 7, respectively.

In Sect. 8 we describe the sophisticated observing techniques used with LABOCA, some of them newly developed. The instrument performance on the sky is described in Sect. 9, along with information on sensitivity, beam shape and noise behavior.

The reduction of the data is performed using a new data reduction software included in the delivery of LABOCA as facility instrument, the BoA (Bolometer array data Analysis) data reduction software package. An account of on-line and off-line data reduction is given in Sect. 10.

Some of the exciting science results already obtained with LABOCA or expected in the near future are outlined in Sect. 11. Our plans for LABOCA's future are briefly presented in Sect. 12.

\section{Tertiary optics}

\subsection{Design and optimization}

The very restricted space in the Cassegrain cabin of APEX and a common first mirror (M3) with the APEX SZ Camera (ASZCa, Schwan et al. 2003) introduced many boundary conditions into the optical design. Eventually, with the help of the ZEMAX ${ }^{3}$ optical design program, a satisfactory solution was found, featuring three aspherical off-axis mirrors (M3, M5, M7), two plane mirrors (M4, M6) and an aspherical lens acting as the entrance window of the cryostat (see Fig. 3). Meeting the spatial constraints, without sacrificing optical quality, is facilitated considerably by the addition of plane mirrors. The design of the optics was made at the MPIfR in coordination with N. Halverson ${ }^{4}$ with respect to sharing the large M3 mirror with the ASZCa experiment.

The maximum possible field diameter of APEX, as limited by the diameter of the Cassegrain hole of the telescope's primary, is about 0.5 degrees. LABOCA, with its 295 close-packed fully efficient horns, covers an almost circular field of view (hereafter $\mathrm{FoV}$ ) of about 0.2 degrees in diameter (or about 100 square arcminutes). The task of the tertiary optics is to transform the $f$-ratio from $f / 8$ at the Cassegrain focus to $f / 1.5$ at the horn array, while correcting the aberrations over the whole FoV of LABOCA under the constraint of parallel output beams. The final design is diffraction limited even for $350 \mu \mathrm{m}$ wavelength, the Strehl ratio is better than 0.994 and the maximum distortion at the focal plane is less than $10 \%$ over the entire FoV (see also Fig. 10).

\footnotetext{
3 http://www . zemax . com/

4 Formerly at University of California at Berkeley, now at University of Colorado, Boulder, USA.
} 

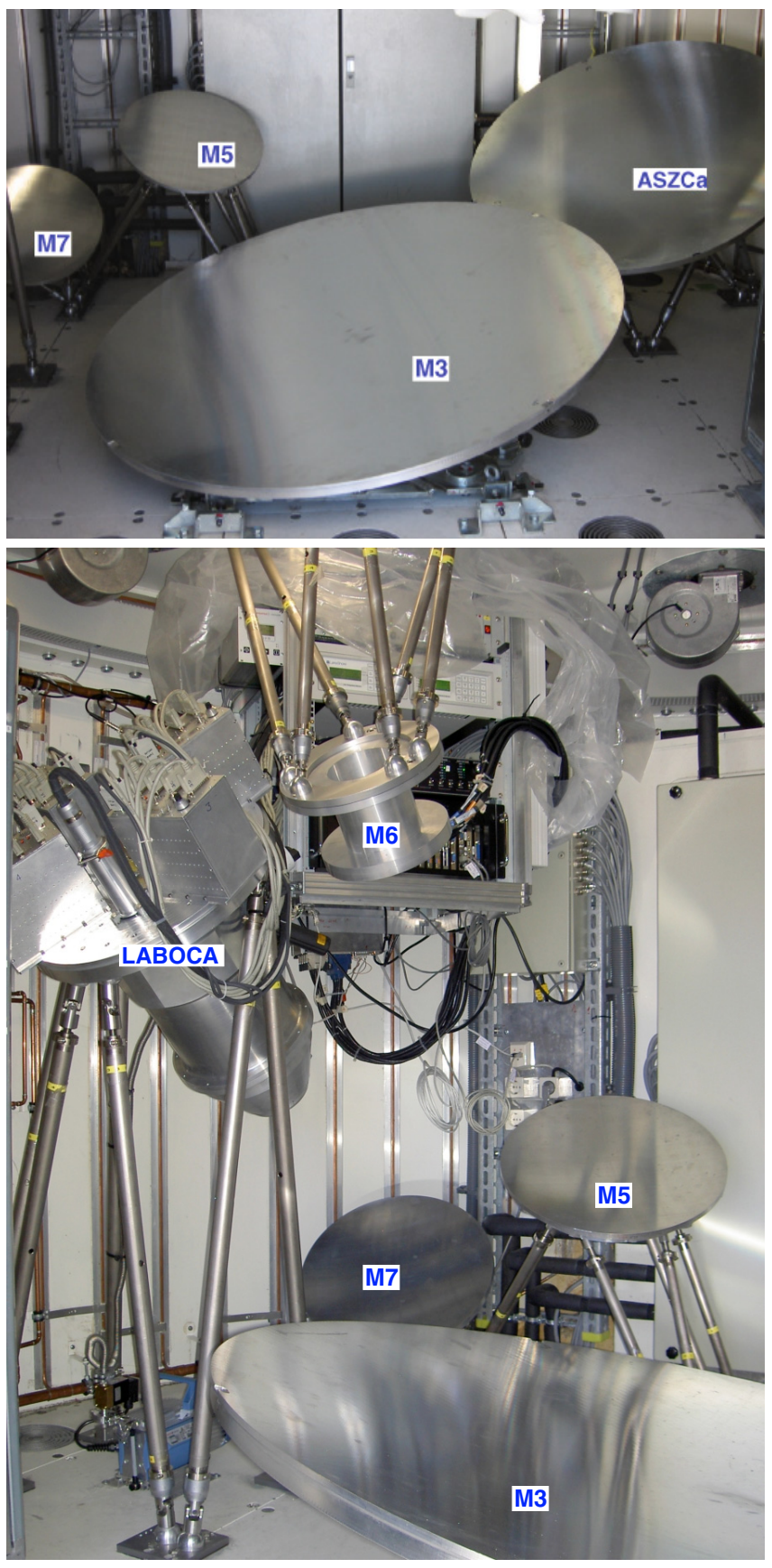

Fig. 4. Top: the mirrors affixed to the floor of the Cassegrain cabin of APEX. Left to right: M7, M5, M3 and one of the mirrors of the ASZCa experiment. In this picture, mirror M3 is positioned for ASZCa. Bottom: the receiver, M3 in the position for LABOCA, M5, M6 and M7. See also Fig. 3.

\subsection{Manufacture}

Mirror M3, an off-axis paraboloid, has been manufactured by a machine shop at the Lawrence Berkeley National Laboratory (LBNL, Berkeley, CA, USA) and is common to both LABOCA and the ASZCa experiment. M3 has a diameter of $1.6 \mathrm{~m}$ and a surface accuracy of $18 \mu \mathrm{m}$ rms but LABOCA uses only the inner $80 \mathrm{~cm}$ disk. It is attached to a bearing on the floor of the Cassegrain cabin, aligned to the optical axis of APEX. Operators can manually rotate the mirror in order to direct the telescope beam to LABOCA or to ASZCa alternatively (see Fig. 4).
Mirrors M4 and M6 are flat, have a diameter of $42 \mathrm{~cm}$ and $26 \mathrm{~cm}$, respectively (manufactured by Kugler ${ }^{5}$, Salem, Germany). They are of optical quality and are both affixed to the ceiling of the cabin. In a near future, mirror M6 will be replaced by the reflection-type half-wave plate of the PolKa polarimeter (Siringo et al. 2004).

Mirrors M5 and M7 are off-axis aspherics, both $50 \mathrm{~cm}$ in diameter, and are affixed to the floor of the cabin. They have been designed and manufactured at the MPIfR and have a surface accuracy of 7 and $5 \mu \mathrm{m}$ rms respectively.

\subsection{Installation and alignment}

All the mirrors of LABOCA (with exception of M3) and the receiver itself are mounted on hexapod positioners (see Fig. 4) provided by VERTEX Antennentechnik ${ }^{6}$ (Duisburg, Germany). Each hexapod is made of an octahedral assembly of struts and has six degrees of freedom $(x, y, z$, pitch, roll and yaw). The lengths of the six independent legs can be changed to position and orient the platform on which the mirror is mounted. VERTEX provided a software for calculating the required leg extensions for a given position and orientation of the platforms. A first geometrical alignment was performed during the first week of September 2006, using a double-beam laser on the optical axis of the telescope and plane replacement mirrors in place of the two active mirrors M5 and M7. The alignment has been checked using the bolometers and hot targets (made of absorbing $^{7}$ material) at different places along the beam, starting at the focal plane and following the path through all the reflections up to the receiver's window. The alignment has been furthermore verified and improved in February 2008.

\section{Cryogenics}

\subsection{Cryostat}

The bolometer array of LABOCA is designed to be operated at a temperature lower than $300 \mathrm{mK}$. This temperature is provided by a cryogenic system made of a wet cryostat, using liquid nitrogen and liquid helium, in combination with a two-stage sorption cooler. A commercial 8-inch cryostat, built by Infrared Labs ${ }^{8}$ (Tucson, AZ, USA), has been customized at the MPIfR to accommodate the double-stage sorption cooler, the bolometer array, cold optics and cold electronics. A high vacuum in the cryostat is provided by an integrated turbomolecular pump backed by a diaphragm pump. Operational vacuum is reached in one single day of pumping.

The cryostat incorporates a 3-liter reservoir of liquid nitrogen and a 5-liter reservoir of liquid helium. After producing high-vacuum $\left(\sim 10^{-6}\right.$ mbar $)$, the cryostat is filled with the liquid cryogens. The liquid nitrogen is used to provide thermal shielding at $77 \mathrm{~K}$ in our labs in Bonn (standard air pressure, $1013 \mathrm{mbar}$ ) and at $73.5 \mathrm{~K}$ at APEX (5107 $\mathrm{m}$ above the sea level) where the air pressure is almost one half of the standard one (about 540 mbar).

The liquid helium provides a thermal shielding at $4.2 \mathrm{~K}$ at standard pressure and $3.7 \mathrm{~K}$ at the APEX site. To keep it

\footnotetext{
5 http://www. kugler-precision. com/

6 http://www. vertexant.de/

7 ECCOSORB AN, Emerson \& Cuming, Rundolph, MA, USA, http : //www . eccosorb. com

8 http://www.irlabs. com/
} 
operational, the system must be refilled once per day. The refilling operation requires about $20 \mathrm{~min}$.

\subsection{Sorption cooler}

The cryostat incorporates a commercial two-stage closed-cycle sorption cooler, model SoCool (Duband et al. 2002) manufactured by Air-Liquide ${ }^{9}$ (Sassenage, France). In this device, a ${ }^{4} \mathrm{He}$ sorption cooler is used to liquefy ${ }^{3} \mathrm{He}$ gas in the adjacent, thermally coupled, ${ }^{3} \mathrm{He}$ cooler. The condensed liquid ${ }^{3} \mathrm{He}$ is then sorption pumped to reach temperatures as low as $250 \mathrm{mK}$, in the absence of a thermal load. Therefore, the double stage design makes it possible to cool the bolometer array down to a temperature lower than $300 \mathrm{mK}$ starting from the temperature of the liquid helium bath at atmospheric pressure. This makes the maintenance of the system much simpler than that of other systems, where pumping on the liquid helium bath is required. The two sorption coolers are closed systems, which means they do not require any refilling of gas and can be operated from the outside of the cryostat, simply by applying electrical power.

To keep the bolometers at operation temperature, the sorption cooler needs to be recycled. The recycling is done by application of a sequence of voltages to the electric lines connected to thermal switches and heaters integrated in the sorption cooler. A typical recycling procedure requires about two hours and can be done manually or in a fully automatic way controlled by the frontend computer (see Sect. 7.4). At the end of the recycling process, both gases, ${ }^{4} \mathrm{He}$ and ${ }^{3} \mathrm{He}$, have been liquefied and the controlled evaporation of the two liquids provides a stable temperature for many hours. After the recycling of the sorption cooler, the bolometer array reaches $285 \mathrm{mK}$. The hold time of the cooler, usually between 10 and $12 \mathrm{~h}$, strongly depends on the parameters used during the recycling procedure. The end temperature is a function of elevation and can be affected by telescope movements, leading to temperature fluctuations $\$ 500 \mu \mathrm{K}$ within one scan, during regular observations, and $\$ 3 \mathrm{mK}$ for wide elevation turns (e.g. during skydips, see Sect. 8.2.3).

\subsection{Temperature monitor}

The cryostat of LABOCA incorporates 8 thermometers to measure the temperature at the different stages: liquid nitrogen, liquid helium, the two sorption pumps, the two thermal switches, evaporator of the ${ }^{4} \mathrm{He}$ and evaporator of the ${ }^{3} \mathrm{He}$. Two LS2 $18^{10}$ devices (Lake Shore Inc., Westerville, OH, USA) are used to monitor the thermometers and to apply the individual temperature calibrations in real-time. The temperature of the ${ }^{3} \mathrm{He}$ stage is measured with higher accuracy with the use of a resistance bridge AVS- $47^{11}$ (Picowatt, Vantaa, Finland), with an error of $\pm 5 \mu \mathrm{K}$. Control and monitor of the cryogenic equipment can be done remotely via the frontend computer (see Sect. 7.4).

\section{Cold optics}

\subsection{Passband definition}

Inside the cryostat, a set of cold filters, mounted on the liquid nitrogen and liquid helium shields define the spectral passband, centered at a wavelength of $870 \mu \mathrm{m}(345 \mathrm{GHz})$ and about

\footnotetext{
${ }^{9}$ http://www.airliquide.com/

10 http://www. lakeshore.com/temp/mn/218po.html

11 http://www.picowatt.fi/avs47/avs47.html
}

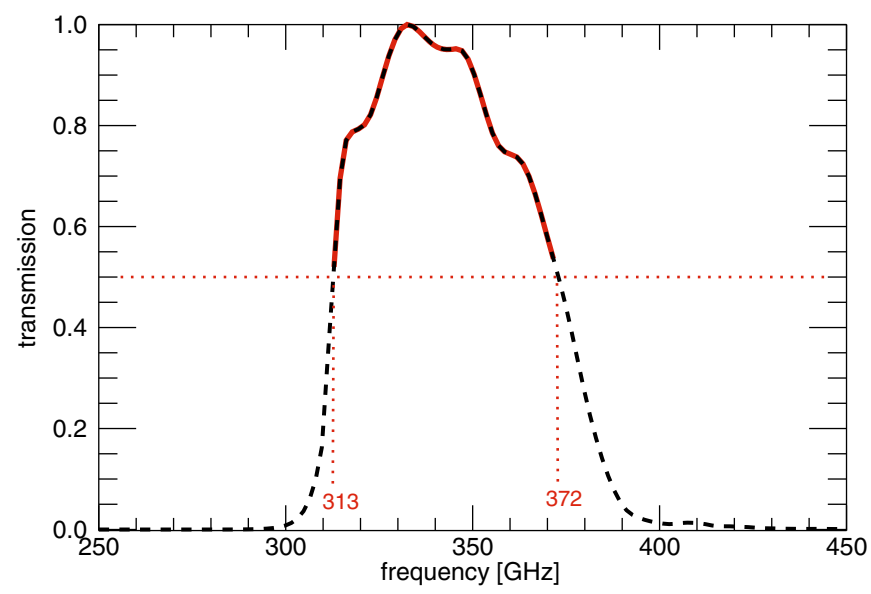

Fig. 5. Spectral response of LABOCA, relative to the maximum. The central frequency is $345 \mathrm{GHz}$, the portion with $50 \%$ or more transmission is between 313 and $372 \mathrm{GHz}$.

$150 \mu \mathrm{m}(60 \mathrm{GHz})$ wide (see Fig. 5). The filters have been designed and manufactured at MPIfR in collaboration with the group of V. Hansen (Theoretische Elektrotechnik ${ }^{12}$, Bergische Universität Wuppertal, Germany) who provided theoretical support and electromagnetic simulations. The passband is formed by an interference filter made of inductive and capacitive meshes embedded in polypropylene. The low frequency edge of the band is defined by the cut-off of the cylindrical waveguide of each horn antenna (see also Sect. 4.2). A freestanding inductive mesh behind the window-lens provides shielding against radio interference.

\subsection{Horn array}

A monolithic array of conical horn antennas, placed in front of the bolometer wafer, concentrates the radiation onto the bolometers. 295 conical horns have been machined into a single aluminum block by the MPIfR machine shop. In combination with the tertiary optics, the horn antennas are optimized for coupling to the telescope's main beam at a wavelength of $870 \mu \mathrm{m}$. The grid constant of the hexagonal array is $4.00 \mathrm{~mm}$. Each horn antenna feeds into a circular wave guide with a diameter of $0.54 \mathrm{~mm}$, acting as a high-pass filter.

\section{Detector}

\subsection{Array design and manufacture}

The bolometer array of LABOCA is made of 295 composite bolometers arranged in an hexagonal layout consisting of a center channel and 9 concentric hexagons (see Figs. 1 and 6). The array is manufactured on a 4-inch silicon wafer coated on both sides with a silicon-nitride film by thermal chemical vapor deposition. On one side of the wafer, 295 squares are structured into the silicon-nitride film used as a mask for the alkaline $\mathrm{KOH}$ etching of the silicon, producing freestanding, unstructured siliconnitride membranes, only $400 \mathrm{~nm}$ thick (see the center picture in Fig. 6). On the other side, the wiring is created by microlithography of niobium and gold thin metal layers. The bolometer array is mounted inside a gold-coated copper ring and is supported by about 360 gold bonding thin wires (see Fig. 6, left), providing the required electrical and thermal connection. This copper ring

\footnotetext{
12 http://www.tet. uni-wuppertal.de/
} 

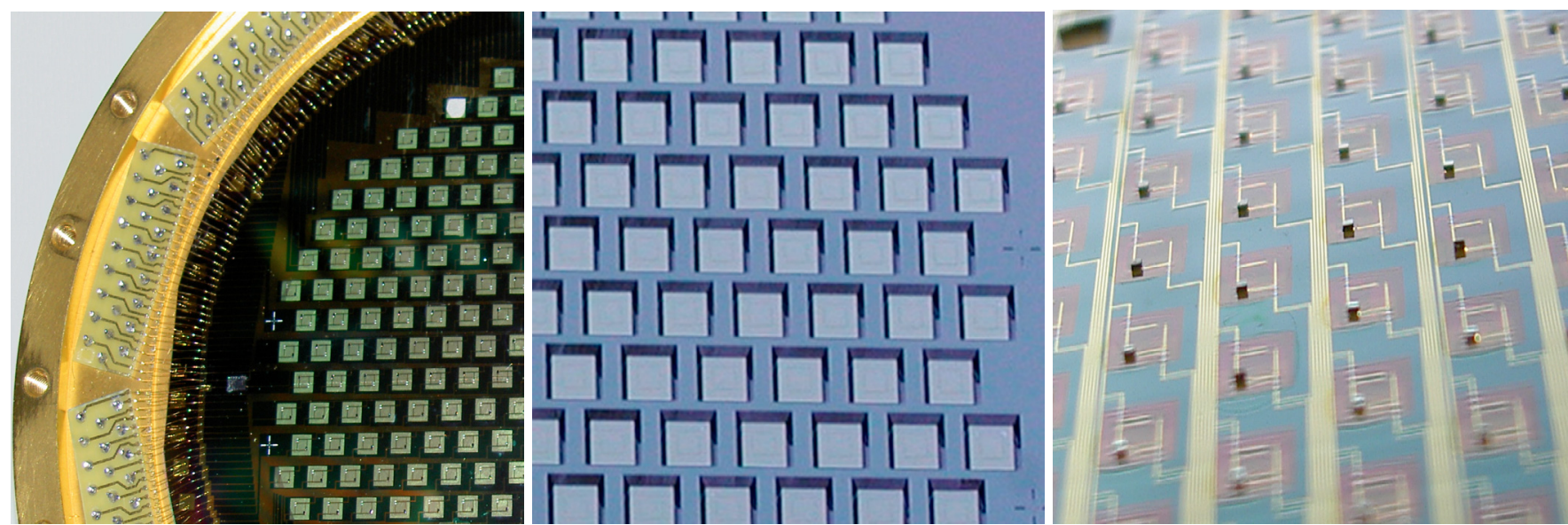

Fig. 6. Pictures of the bolometer array of LABOCA. Left: a detail of the array mounted in its copper ring. Some bonding wires are visible. Center: the side of the array where the bolometer cavities are etched in the silicon wafer. Right: wiring side of the array. The thermistors are visible as small cubes on the membranes. One broken membrane is visible on the top left corner. See also Fig. 1.

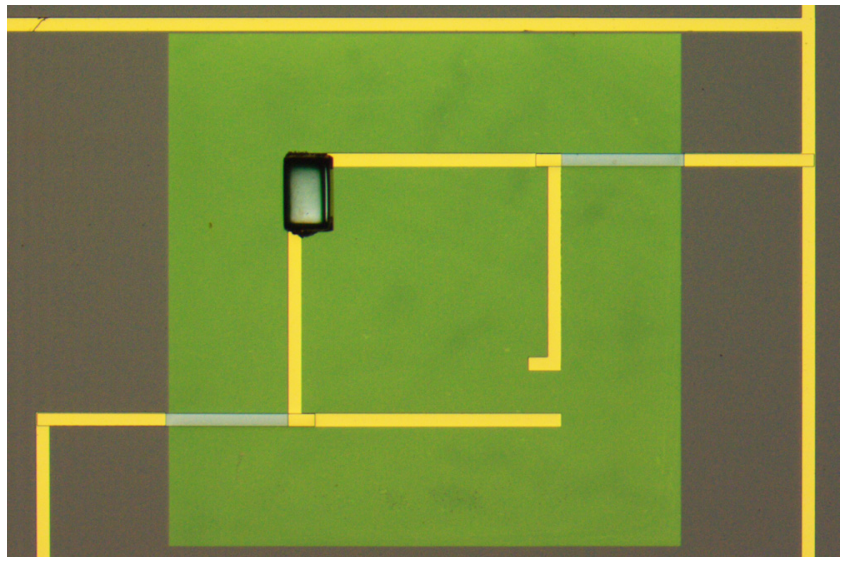

Fig. 7. Wiring side of one bolometer of LABOCA, seen under a microscope. The membrane is the green colored area. The black box is the NTD thermistor. The wiring is made of gold (yellow) and niobium (gray) thin metal layers. See also Fig. 6.

also serves as a mount for the backshort reflector, at $\lambda / 4$ distance from the array, and 12 printed circuit boards hosting the load resistors and the first electronic circuitry (see Sect. 6).

\subsection{Bolometer design and manufacture}

The description of a composite bolometer can be simplified as the combination of two elements: an extremely sensitive temperature sensor, called thermistor, and a radiation absorber. In the bolometers of LABOCA, the absorbing element is made of a thin film of titanium deposited on the unstructured silicon-nitride membranes. LABOCA uses neutron-transmutation doped germanium semiconducting chips (NTD, Haller et al. 1982) as thermistors. The NTD thermistors are made from ultra-pure germanium doped by neutron-transmutation in a nuclear reactor. The thermistors, needed to measure the temperature of the absorber, are soldered on the bolometer membranes to gold pads connected to the outer edge of the silicon wafer through a pair of patterned niobium wires, superconducting at the operation temperature (see Figs. 7 and 6, right). The soldering of the thermistors was the only manual step in the manufacture of the bolometers. LABOCA has so-called flatpack NTD thermistors, which have two ion implanted and metalized contacts on one side of the thermistor block. The chips are optimized to work at a temperature lower than $300 \mathrm{mK}$, where they show an electric impedance in the range of 1 to $10 \mathrm{MOhms}$.

\section{Cold electronics}

\subsection{Bias resistors}

Given the high impedance of the NTD thermistors, the electric scheme of the first bolometer circuitry requires very high impedance load resistors, which are needed to current bias the bolometers. These bias resistors are 312 identical $30 \mathrm{MOhm}$ chips, made of a nichrome thin film deposited on silicon substrate (model MSHR-4 produced by Mini-Systems Inc. ${ }^{13}, \mathrm{~N}$. Attleboro, MA, USA) mounted on 12 identical printed circuit boards, to form 12 groups of 26 resistors. This configuration is reflected in the following distribution of the bolometer signals. The circuit boards are mounted on the same copper ring which holds the bolometer array (see Sect. 5.1) and electrically connected to the bolometers through miniature RF filters.

\subsection{Junction field effect transistors (JFETs) source followers}

The high impedance of the bolometers makes the system sensitive to microphonic noise pickup, therefore JFETs (Toshiba 2SK369) are used as source followers in order to decrease the impedance of the electric lines down to a few kOhms before they reach the high gain amplification units at room temperature. Following the wiring scheme of the bias resistors, the JFETs are also in groups of 26 soldered onto 12 printed circuit boards, electrically connected to the corresponding bias resistors by 12 flat cables made of manganin traces embedded in Kapton $^{14}$ (manufactured by VAAS Leiterplattentechnologie ${ }^{15}$, Schwäbisch-Gmünd, Germany), thermally shunted to the liquid helium tank. The 12 JFET boards are assembled in groups of three into four gold-coated copper boxes, thermally connected to the liquid nitrogen tank. Inside each box, during regular operation, the 78 JFETs are self-heated to a temperature of about $110 \mathrm{~K}$, where they show a minimum of their intrinsic noise. Through the connections in the JFET boxes, the wiring scheme

\footnotetext{
13 http://www . mini-systemsinc.com/

14 DuPont, http://www2 . dupont . com/Kapton/en_US/

15 http://www . vaas-1t.de/
} 
of 12 groups of 26 channels is translated to a new scheme of 4 groups of 80 channels.

\section{Warm electronics and signal processing}

\subsection{Amplifiers}

The 312 channels exiting the cryostat of LABOCA are distributed to 4 identical, custom made, amplification units, providing 80 channels each. Of these, 295 are bolometers, 17 are connected to $1 \mathrm{MOhm}$ resistors mounted on the bolometer ring (used for technical purposes, like noise monitoring and calibrations) and the remaining 8 are not connected. Each amplification unit is made of 16 identical printed circuit boards and each board provides 5 low noise, high gain amplifiers. Each unit also includes a low noise battery used to generate the bias voltage and the circuitry to produce the AC biasing and perform real time demodulation of the 320 signals. The AC bias reference frequency is not internally generated but is provided from the outside, thus allowing synchronization of the biasing to an external frequency source.

Each amplification unit is equipped with a digital interface controlled by a microprocessor programmed to provide remote control of the amplification gain and of the DC offset removal procedure. This is required because LABOCA is a total power receiver and the signals carry a floating DC offset which could exceed the dynamic range of the data acquisition system. To avoid saturation, therefore, the DC offsets are measured and subtracted from the signals at the beginning of every integration. The values of the 320 offsets are temporarily stored in a local memory and, at the end of the observation, are written into the corresponding data file for use in the data reduction process. The digital lines use the $\mathrm{I}^{2} \mathrm{C}$ protocol ${ }^{16}$ and are accessible remotely via the local network through $\mathrm{I}^{2} \mathrm{C}$-to-RS232 $2^{17}$ interfaces controlled by the frontend computer (see Sect. 7.4). The amplification gain can be set in the range from 270 to 17280 .

\subsection{Data acquisition}

The 320 output signals from the 4 amplification units are digitized over 16 bits by 4 multifunction data acquisition (DAQ) boards (National Instruments $\left.{ }^{18} \mathrm{M}-6225-\mathrm{PCI}\right)$, providing 80 analog inputs each and synchronized to the same sample clock by a $\operatorname{RTSI}^{19}$ bus . The maximum data sampling is $2500 \mathrm{~Hz}$ and the dynamic range can be selected over 5 predefined ranges. The four boards provide also 24 digital input/output lines each, some of them used for the generation of the bias reference frequency and to monitor the digital reference signals (sync/blank) of the wobbler. For the time synchronization of the data to the APEX control software (APECS, Muders et al. 2006) the data acquisition system is equipped with a precision time interface (PCISyncClock $32^{20}$ from Brandywine Communications, Tustin, CA, USA) synchronized to the station GPS clock via IRIG-B ${ }^{21}$ time code signal.

The AC bias reference frequency is provided by the data acquisition system as a submultiple of the sampling frequency, thus

\footnotetext{
16 Inter-Integrated Circuit, a serial bus to connect hardware devices.

17 Recommended Standard 232, a standard for serial binary data communication.

18 http://www . ni.com/

19 Real-Time System Integration, a bus used to share and exchange timing and control signals between multiple boards.

20 http: //www . brandywinecomm. com

21 Inter Range Instrumentation Group, standardized time code format.
}

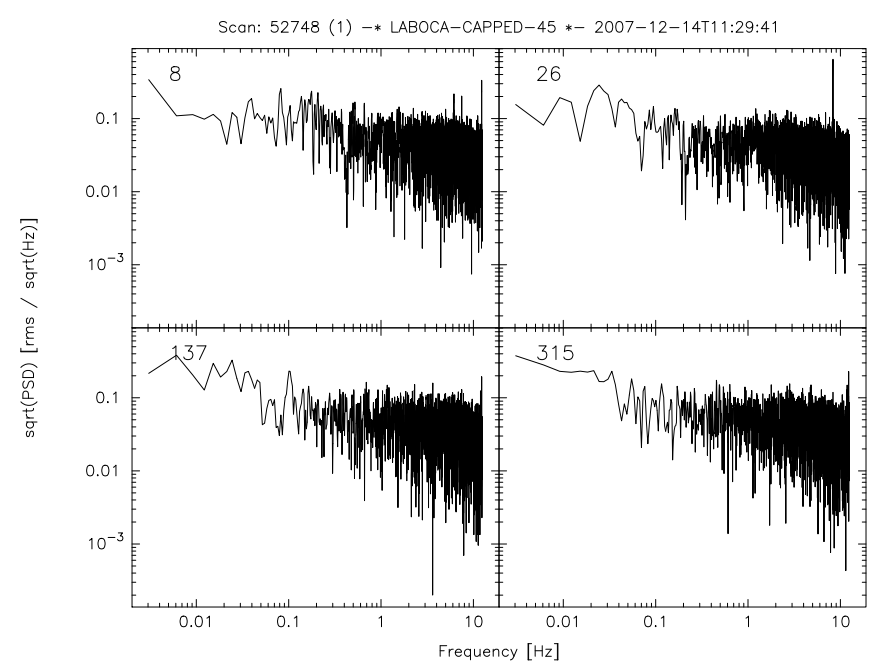

Fig. 8. Noise spectra for selected bolometers on a room temperature absorber (capped optics). The signals, sampled at $1 \mathrm{kHz}$, were downsampled in real-time to $25 \mathrm{~Hz}$ by the bridge computer. The spectra are free of microphonic pick-ups and show the $1 / f$ noise onset at $\sim 0.1 \mathrm{~Hz}$.

synchronizing the bias to the data sampling. Typical values used for observations are $1 \mathrm{kHz}$ for the sampling rate and $333 \mathrm{~Hz}$ for the AC bias. The backend computer has two network adapters: one is connected to the local area network, the other one is exclusively used for the output data stream and is connected in a private direct network with the bridge computer (see also Sect. 7.3).

The data acquisition software is entirely written using LabVIEW $^{22}$ (National Instruments). The drivers for the data acquisition hardware are provided by the NI-DAQmx ${ }^{23}$ package. Custom drivers for LabVIEW have been developed to access the GPS clock interface. The backend software runs a server to stream the output data to the bridge computer and allows remote control and monitoring of the operation via a CORBA ${ }^{24}$ object interfaced to the APECS through the local area network.

\subsection{Anti-aliasing filtering and downsampling}

The amplification units of LABOCA use the bias signal, which is a square waveform, as reference to operate real-time demodulation of the AC-biased bolometer signals. Therefore, all the frequencies present in the bolometer readout lines end up aliased around the odd-numbered harmonics of the bias frequency. Microphonics pickup by the high-impedance bolometers at a few resonant frequencies can produce a forest of lines in the final readout, polluting even the lower part of the postdetection frequency band, where the astronomical signals are expected. To overcome this, we introduced an intermediate stage into the sampling scheme, the so-called bridge computer. The bolometer signals, acquired by the backend at a relatively high sampling rate (usually $1 \mathrm{kHz}$ ), well above the rolling-off of the anti-alias filters embedded in the amplifiers, are sent to the bridge computer where they are digitally low-pass filtered and then downsampled to a much lower rate (usually $25 \mathrm{~Hz}$ ), more appropriate for the astronomical signals produced at the typical scanning speeds (see also Sect. 8). The digital real-time

\footnotetext{
22 http://www.ni.com/labview/

${ }^{23}$ http://www.ni.com/dataacquisition/nidaqmx.htm

${ }^{24}$ Common Object Request Broker Architecture, a set of standards which define the protocol for interaction between the objects of a distributed system.
} 
anti-alias filtering and downsampling is performed by a nonrecursive convolution filter with a Nutall window such that its rejection at the Nyquist frequency is $\sim 3 \mathrm{~dB}$ and falls steeply to $\sim 100 \mathrm{~dB}$ soon beyond that. The bias reference frequency was accordingly selected to maximize the astronomically useful post-detection bandwidth. The resulting bolometer signals are generally white between $0.1-12.5 \mathrm{~Hz}$ and free of unwanted microphonic interference (see Fig. 8).

To seamlessly integrate the bridged readout into the APEX control system, the bridge computer also acts as a fully functional virtual backend that forwards all communication between the actual backend computer and the control system, while intercepting and reinterpreting any commands of interest for the downsampling scheme.

\subsection{Frontend computer}

The so-called frontend computer communicates with the hardware of LABOCA through the local area network. It is devoted to monitor and control most of the electronics of the instrument (e.g. monitoring of all the temperature stages, control of the sorption cooler, calibration unit, power lines...) and also provides a CORBA object for interfacing to APECS, allowing remote operation of the system. The frontend software is entirely written using LabVIEW and custom drivers have been developed for some hardware devices embedded in LABOCA.

\section{Observing modes}

\subsection{Mapping modes}

In order to reach the best signal-to-noise ratio using the fast scanning technique (Reichertz et al. 2001) with LABOCA, the frequencies of the signal produced by scanning across the source need to match the white noise part of the post-detection frequency band $(0.1-12.5 \mathrm{~Hz}$, see Fig. 8), mostly above the frequencies of the atmospheric fluctuations. Thus, with the $\sim 19^{\prime \prime}$ beam (see Sect. 9.3), the maximum practical telescope scanning speed for LABOCA is about $4^{\prime} / \mathrm{s}$. This is also the limiting value to guarantee the required accuracy in the telescope position information of each sample. The minimum scanning speed required for a sufficient source modulation depends on the atmospheric stability and on the source structure and is typically about $30^{\prime \prime} / \mathrm{s}$. The APEX control system currently supports two basic scanning modes: on-the-fly maps (OTF) and spiral scanning patterns.

\subsubsection{Spirals}

Spirals are done with a constant angular speed and an increasing radius, therefore the linear scanning velocity is not constant but increases with time. We have selected two spiral modes of $20 \mathrm{~s}$ and $35 \mathrm{~s}$ integration time, both producing fully sampled maps of the whole FoV with scanning velocities limited between $1^{\prime} / \mathrm{s}$ and $2.5 /$ s. The spiral patterns are kept compact (maximum radius $\lesssim 2^{\prime}$ ), the scanned area on the sky is only slightly larger than the FoV and most of the integration time is spent on the central $11^{\prime}$ of the array. These spirals are the preferred observing modes for pointing scans on sources with flux densities down to a few Jy.

For fainter sources, the basic spiral pattern can be combined with a raster mapping mode (raster-spirals) on a grid of pointing positions resulting in an even denser sampling of the maps and longer integration time (see Fig. 9, top panel). These compact raster-spirals give excellent results for sources smaller than the
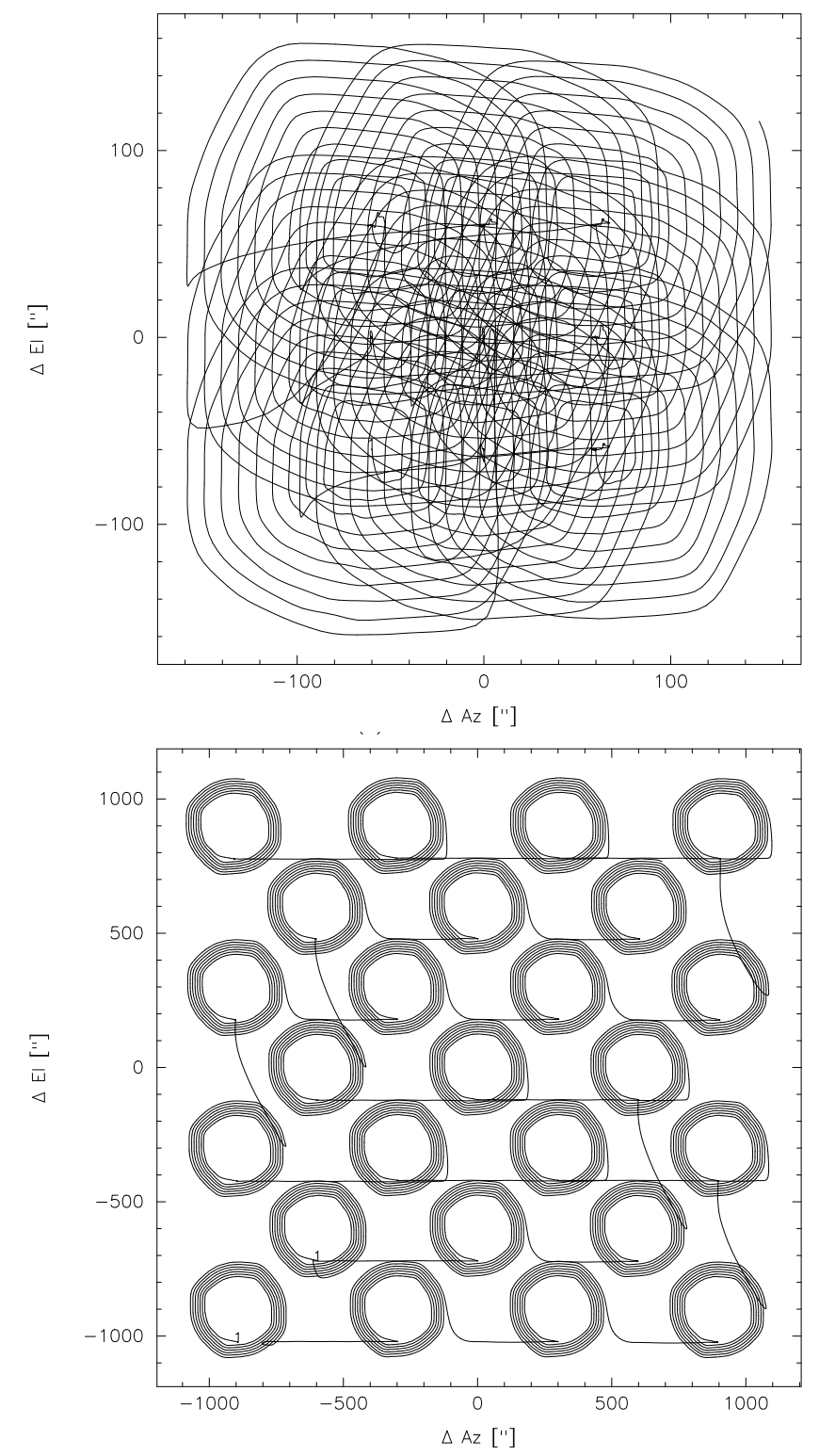

Fig. 9. Examples of raster-spiral patterns. The two plots show the scanning pattern of the central beam of the array in horizontal coordinates. The compact pattern shown in the top panel is optimized to map the field of view of LABOCA. The large scale map shown in the bottom panel consists of 25 raster positions and covers a field of $0.5 \times 0.5$ degrees. The complete scan required $19 \mathrm{~min}$ and the final map covers about $2000 \times 2000$ arcsec with uniform residual rms noise.

FoV of LABOCA and are also suitable for integrations of very faint sources.

The flexibility in the choice of the spiral parameters also allows spiral observing patterns to be used to map fields much larger than the FoV. The bottom panel of Fig. 9 shows an example of raster of spirals optimized to give an homogeneous coverage across a field of $0.5 \times 0.5$ degrees. In this case, the spirals start with a large radius and follows an almost circular scanning pattern for each raster position. This mapping mode is very useful for cosmological deep field surveys since co-adding several such raster-spiral scans, taken at different times and thus at different orientations, provides an optimal compromise between telescope overheads, uniform coverage and cross-linking of individual map positions (see Kovács 2008). 


\subsubsection{On-the-fly maps (OTF)}

OTF scans are rectangular scanning patterns produced moving back-and-forth along alternating rows with a linear constant speed and accelerating only at the turnarounds. They can be performed in horizontal or equatorial coordinates and the scanning direction can be rotated relatively to the base system for both coordinate systems. OTF patterns have been tested for maps on the scales of the FoV up to long slews across the plane of the Milky Way (2 degrees). Small cross-linked OTFs (of size $\sim$ FoV of LABOCA) give results comparable to the raster-spirals (Kovács 2008), but the overheads are much larger at a scanning speed of $2^{\prime}$ /s. For larger OTFs the relative overheads decrease.

\subsection{Ancillary modes}

\subsubsection{Point}

The standard pointing procedure consists of one subscan in spiral observing mode and results in a fully sampled map of the FoV of LABOCA. The pointing offsets relative to the pointing model are computed via a two-dimensional Gaussian fit to the source position in the map using a BoA pipeline script (see Sect. 10). Note that this pointing procedure is not limited to pointing scans of the central channel of the array but works independently of the reference bolometer, thus allowing pointing scans centered on the most sensitive part of the array.

\subsubsection{Focus}

The default focusing procedure is made of 10 subscans at 5 different subreflector positions and $5 \mathrm{~s}$ of integration time each. This is the only observing mode without scanning telescope motion. As a result, we are currently restricted to sources brighter than the atmospheric variations (Mars, Venus, Saturn and Jupiter). However, initial tests confirmed that using the wobbler to modulate the source signal allows focusing on weaker sources, too. This is the only observing mode, so far, for which the use of the wobbler with LABOCA has been tested.

\subsubsection{Skydips}

The attenuation of the astronomical signals due to the atmospheric opacity is determined with skydips. These scans measure the power of the atmospheric emission as a function of the airmass while tipping the telescope from high to low elevation. A skydip procedure consists of two steps: a hot-sky calibration scan, to provide an absolute measurement of the sky temperature, followed by a continuous tip scan in elevation; see also Sect. 9.5.

\section{Performance on the sky and sensitivity}

\subsection{Number of channels}

At the time of the commissioning, the number of channels with sky response was 266 (90\% of the nominal 295 bolometers). Of these, 6 channels show cross-talk and 12 channels have low sensitivity (less than $10 \%$ of the mean responsivity). Two additional bolometers have been blinded by blocking their horn antennas with absorber material so they can be used to monitor the temperature fluctuations of the array. The number of channels used for astronomical observations is therefore 248 (84\% yield, see Fig. 10).

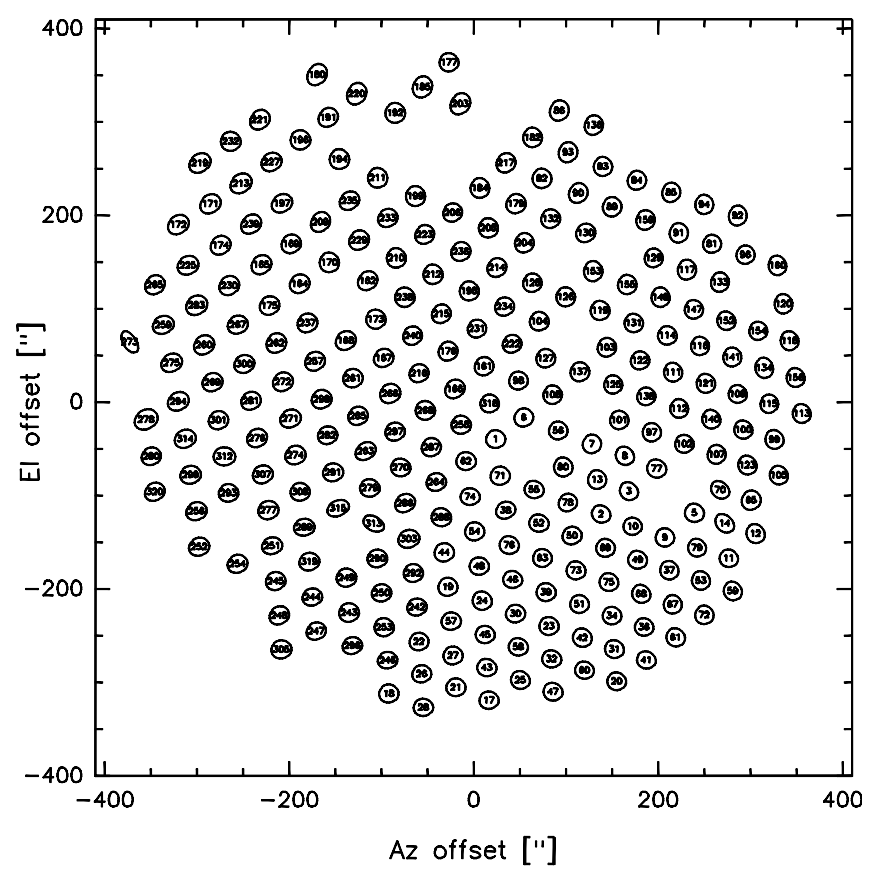

Fig. 10. Footprint of LABOCA on sky, measured with a beam map on the planet Mars. The ellipses represent the $F W H M$ shape of each beam on sky, as given by a two-dimensional Gaussian fit to the single-channel map of each bolometer. Only bolometers with useful signal-to-noise ratios are shown in this map. See also Fig. 12.

\subsection{Array parameters}

Position on sky and relative gain of each bolometer are derived from fully sampled maps (hereafter called beam maps) of the planets Mars and Saturn (see Fig. 10), besides giving a realistic picture of the optical distortions over the FoV. Variations among maps were found to be within a few arc seconds for the positions and below $10 \%$ for peak flux densities. A table with average receiver parameters $(\mathrm{RCP})^{25}$ is periodically computed from beam maps and implemented in the BoA software (see Sect. 10). The accuracy of the relative bolometer positions from this master RCP is typically below 1" (5\% of the beam size) and the gain accuracy is better than $5 \%$, confirming the good quality (small distortion over the entire FoV) of the tertiary optics (see also Sect. 2).

\subsection{Beam shape}

The LABOCA beam shape was derived for individual bolometers from fully sampled maps on Mars (see Fig. 10) as well as on pointing scans on Uranus and Neptune. Both methods lead to comparable results and give an almost circular Gaussian with a $F W H M$ of $19.2 \pm 0.7$ (after deconvolution from the source and pixel sizes). We also investigated the error beam pattern of LABOCA on beam maps on Mars and Saturn (see Fig. 11). The beam starts to deviate from a Gaussian at $-20 \mathrm{~dB}(1 \%$ of the peak intensity). The first error beam pattern can be approximated by a Gaussian with a peak of $-18.3 \mathrm{~dB}$ and a $F W H M$ of $70^{\prime \prime} \pm 5^{\prime \prime}$, the support legs of the subreflector are visible at the $-25 \mathrm{~dB}(0.3 \%$ level). The fraction of the power in the first error beam is $\sim 18 \%$.

25 The latest RCP table is available at: http://www. apex-telescope.org/bolometer/laboca/calibration/ LABOCA-centred.rcp 


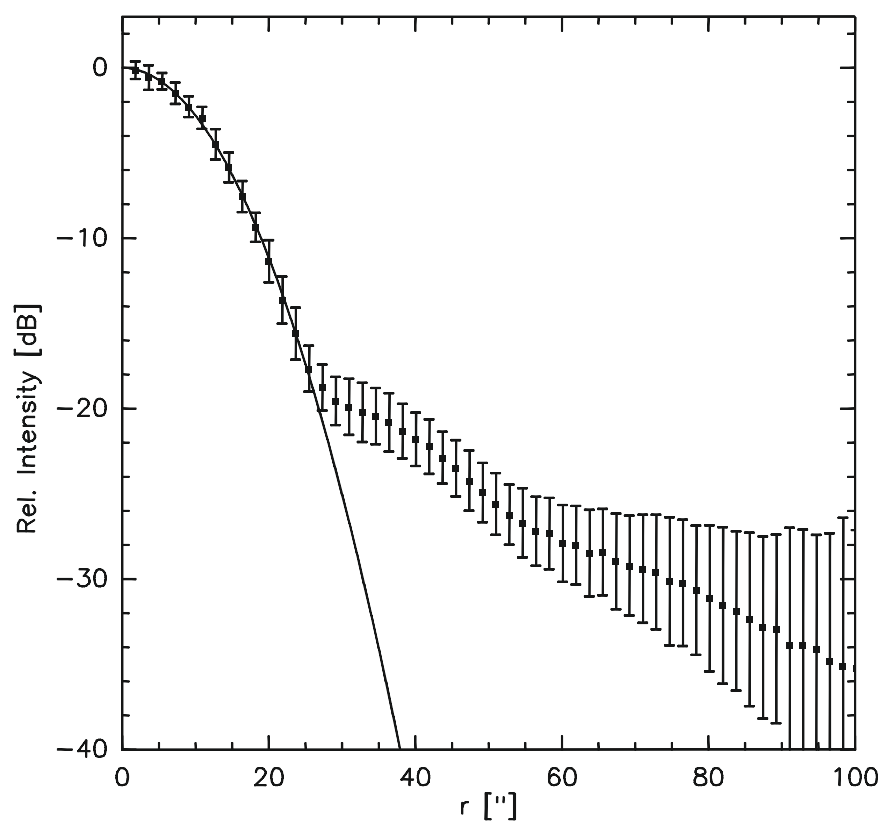

Fig. 11. Radial profile of the LABOCA beam derived by averaging the beams of all 248 functional bolometers from fully sampled maps on Mars. The error bars show the standard deviation. The main beam is well described by a Gaussian with a FWHM of 19 ,. $^{\prime} \pm 0$ "' 7 and starts to deviate at $-20 \mathrm{~dB}$.

\subsection{Calibration}

The astronomical calibration was achieved on Mars, Neptune and Uranus and a constant conversion factor of $6.3 \pm$ $0.5 \mathrm{Jy}_{\text {beam }}^{-1} \mu \mathrm{V}^{-1}$ was determined between LABOCA response and flux density. For the determination of the calibration factor we have used the fluxes of planets determined with the software $\mathrm{ASTRO}^{26}$. The overall calibration accuracy for LABOCA is about $10 \%$. We have also defined a list of secondary calibrators and calibrated them against the planets (see Appendix A). In order to improve the absolute flux determination, the calibrators are observed routinely every $\sim 2 \mathrm{~h}$ between observations of scientific targets.

\subsection{Sky opacity determination}

Atmospheric absorption in the passband of LABOCA attenuates the astronomical signals as $\exp \left(-\tau_{\text {los }}\right)$ where the line of sight optical depth $\tau_{\text {los }}$ can be as high as 1 for observations at low elevation with $2 \mathrm{~mm}$ of precipitable water vapor (PWV), typical limit for observations with LABOCA. The accuracy of the absolute calibration, therefore, strongly depends on the precision in the determination of $\tau_{\text {los }}$.

We use two independent methods for determining $\tau_{\text {los }}$. The first one relies on the PWV level measured every minute by the APEX radiometer broadly along the line of sight. The PWV is converted into $\tau_{\text {los }}$ using an atmospheric transmission model (ATM, Pardo et al. 2001) and the passband of LABOCA (see Sect. 4.1). The accuracy of this approach is limited by the knowledge of the passband, the applicability of the ATM and the accuracy of the radiometer.

The second method uses skydips (see Sect. 8.2.3). As the telescope moves from high to low elevation, $\tau_{\text {los }}$ increases with airmass. The increasing atmospheric load produces an increasing

${ }^{26}$ GILDAS package, http://www.iram.fr/IRAMFR/GILDAS total power signal converted to effective sky temperature ( $\left.T_{\text {eff }}\right)$ by direct comparison with a reference hot load. The dependence of $T_{\text {eff }}$ on elevation is then fitted to determine the zenith opacity $\tau$, used as parameter (see, e.g., Chapman et al. 2004).

Since LABOCA is installed in the Cassegrain cabin of APEX, when performing skydips the receiver suffers a wide, continuous rotation (about 70 degrees in $20 \mathrm{~s}$ ), which affects the stability of the sorption cooler, thus inducing small variations of the bolometers temperature $(\sim 1-2 \mathrm{mK})$. These temperature fluctuations mimic an additional total power signal with amplitude comparable to the atmospheric signal. The bolometers temperature, however, is monitored with high accuracy by the ${ }^{3} \mathrm{He}$-stage thermometer (see Sect. 3.3) and by the two blind bolometers (see Sect. 9.1), making possible a correction of the skydip data.

The values of $\tau$ resulting from the skydip analysis are robust, yet up to $\sim 30 \%$ higher than those obtained from the radiometer. There are several possible explanation for the discrepancy: it could be the result of some incorrect assumptions going into the skydip model (e.g. the sky temperature), or the model itself may be incomplete. The non-linearity of the bolometers can be another factor. The detector responsivities are expected to change with the optical load as $\sim \exp \left(-\gamma \tau_{\text {los }}\right.$ ) (to first order in $\tau$ ), where $\gamma$ can be related to the bolometer constants (Mather 1984) and the optical configuration. Combined with the sky response, the bolometer non-linearities would increase the effective skydip $\tau$ by a factor $(1+\gamma)$.

Our practical approach to reconciling the results obtained with the two methods has been to use a linear combination of radiometer and skydip values such that it gives the most consistent calibrator fluxes at all elevations ${ }^{27}$. The excellent calibration accuracy of LABOCA (see Appendix A) underscores this approach.

\subsection{Sensitivity}

The noise-weighted mean point-source sensitivity of the array (noise-equivalent flux density, NEFD) determined from on-sky integrations, is $55 \mathrm{mJy} \mathrm{s}^{1 / 2}$ (sensitivity per channel). This value is achieved only by filtering the low frequencies (hence large scale emission) to reject residual sky-noise. For extended emission, without low frequency filtering, there is a degradation of sensitivity to a mean array sensitivity of $80-100 \mathrm{mJy} \mathrm{s} \mathrm{s}^{1 / 2}$ depending on sky stability. However, there are significant variations of the sensitivity across the array (see Fig. 12, top).

For detection experiments of compact sources with known position, LABOCA can be centered on the most sensitive part of the array rather than on the geometric center. This results in an improved point source sensitivity of $\sim 40 \mathrm{mJy} \mathrm{s}^{1 / 2}$ for compact mapping pattern like spirals.

\subsection{Mapping speed and time estimate}

The relation between the expected residual rms map noise $\sigma$, the surveyed area on the sky and the integration time can be expressed as

$t_{\text {int }}=\frac{\left(X_{\text {scan }}+D\right)\left(Y_{\text {scan }}+D\right)}{A_{\text {beam }} N_{\text {bol }}}\left(\frac{f_{\text {samp }} N E F D \mathrm{e}^{\tau_{\text {los }}}}{\sigma}\right)^{2}$

\footnotetext{
27 A text file (BoA readable, see Sect. 10) containing the zenith opacities calculated from the skydips and the radiometer is available at: http://www . apex-telescope.org/bolometer/laboca/ calibration/opacity/
} 

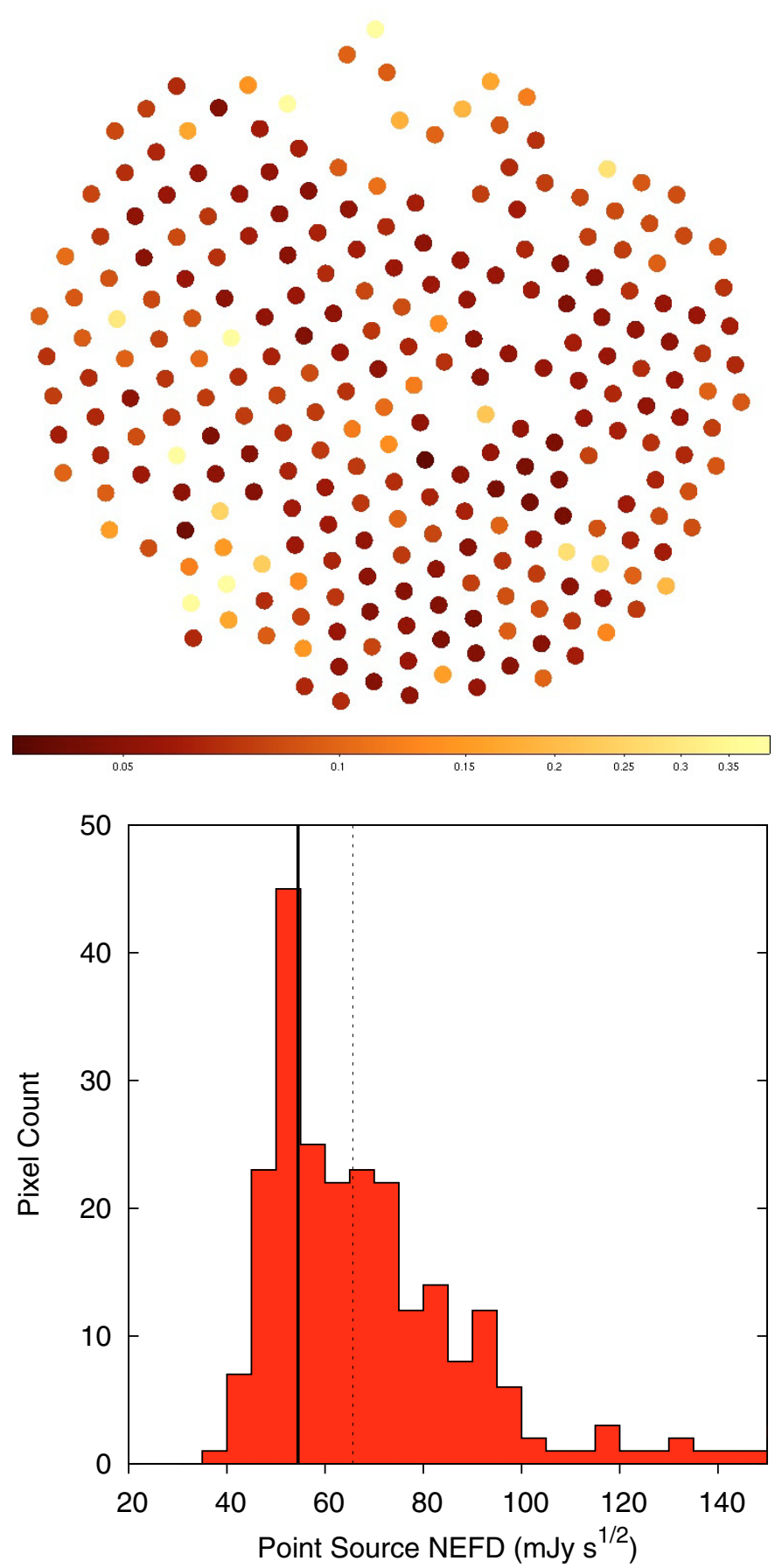

Fig. 12. Top: effective point source sensitivities (after low frequencies filtering) of all the useful bolometers of LABOCA (color scale in $\mathrm{Jy} \mathrm{beam}^{-1}$ ). The positions were determined from beam maps on Mars, thus providing a realistic picture of the optical distortions over the FoV (see also Fig. 10). Bottom: distribution of the effective point source sensitivities on the LABOCA array ( $5 \mathrm{mJy} \mathrm{s}^{1 / 2}$ binning). The total mapping speed of the array is as if the 250 good bolometers were all identical at a level of $54.5 \mathrm{mJy}_{\text {beam }}{ }^{-1} \mathrm{~s}^{1 / 2}$ (thick black line). The median sensitivity is also shown (dotted line).

where $X_{\text {scan }}, Y_{\text {scan }}$ are the dimensions of the area covered by the scanning pattern, $D$ is the size of the array, $A_{\text {beam }}$ is the area of the LABOCA beam, $N_{\text {bol }}$ is the number of working bolometers, $t_{\text {int }}$ is the on source integration time, NEFD is the average array sensitivity, $f_{\text {samp }}$ is the number of grid points per beam and $\tau_{\text {los }}$

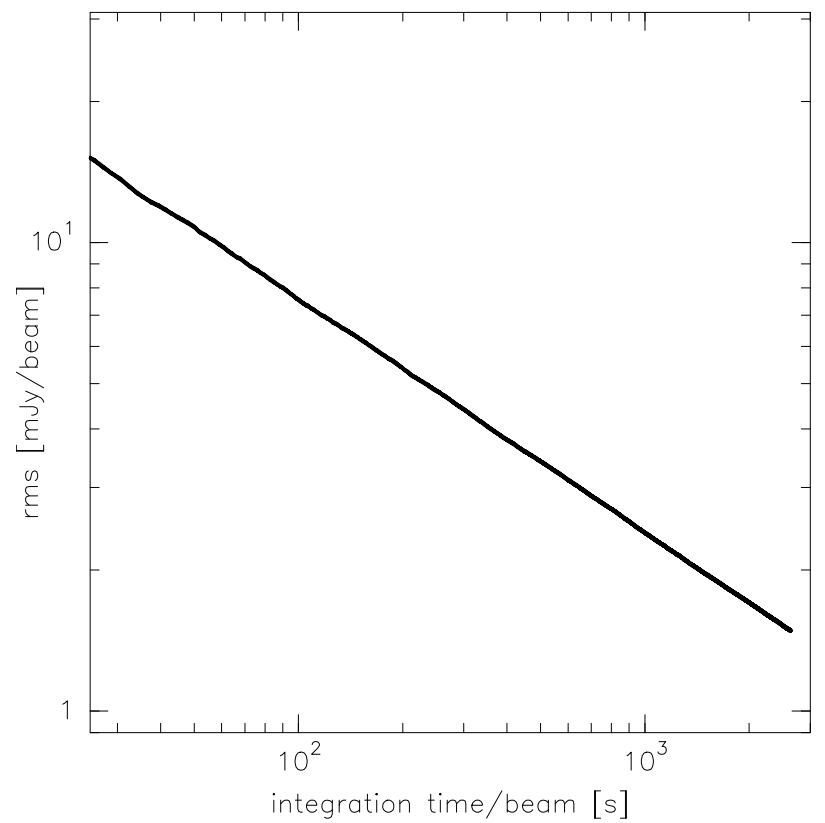

Fig. 13. Noise behavior for deep integrations has been studied by coadding about $350 \mathrm{~h}$ of blank-field data. The plot shows the effective residual rms noise on sky.

the line-of-sight opacity. This formula ${ }^{28}$ does not include sensitivity variations across the array and the increasing sparseness of data points toward the map edges (the latter effect depends on the mapping mode). We have tested this estimate on deep integrations for point like and extended sources and found a reasonable agreement in the measured rms noise of the processed maps.

\subsection{Noise behavior in deep integrations}

In order to study how the noise averages down with increasing integration time, about $350 \mathrm{~h}$ of blank-field mapping observations have been co-added with randomly inverted signs to remove any sources while keeping the noise structure. As shown in Fig. 13, the noise integrates down with $t^{-1 / 2}$, as expected.

\section{Data reduction}

LABOCA data are stored in MB-FITS format (Multi-Beam FITS) by the data writer embedded in APECS. A new software package has been specifically developed to reduce LABOCA data: the Bolometer array data Analysis software (BoA). It is mostly written in the Python language, except for the most computing demanding tasks, which are written in Fortran90.

BoA was first installed and integrated in APECS in early 2006. An extensive description of its functionalities will be given in a separate paper (Schuller et al., in prep.). In this section, we outline the most important features for processing LABOCA data.

\subsection{On-line data reduction}

During the observations carried out at APEX, the on-line data calibrator (as part of APECS) performs a quick data reduction

\footnotetext{
28 An online time estimator is available at: http://www . apex-telescope.org/bolometer/laboca/ obscalc/
} 
of each scan to provide the observer with a quick preview of the maps or spectra being observed. This is of particular importance for the basic pointing and focus observing modes, for which the on-line calibrator computes and sends back to the observer the pointing offsets or focus corrections to be applied. For both focus and pointing scans, only a quick estimate of the correlated noise (see below) is computed and subtracted from the data. The focus correction is derived from a parabolic fit to the peak flux measured by the reference bolometer as a function of the subreflector position. For pointing scans, the signals of all usable channels are combined into a map of the central $5^{\prime} \times 5^{\prime}$ area, in horizontal coordinates. A two-dimensional elliptical Gaussian is then fitted to the source in this map, which gives the pointing offsets, as well as the peak flux and the dimensions of minor and major axis of the source.

\subsection{Off-line data reduction}

The BoA software can also be used off-line to process any kind of bolometer data acquired at APEX. The off-line BoA runs in the interactive environment of the Python language. In a typical off-line data reduction session, several scans can be combined together, for instance to improve the noise level on deep integrations, or to do mosaicing of maps covering adjacent areas. The result of any data reduction can be stored in a FITS file using standard world coordinate system (WCS) keywords, which can then be read by other softwares for further processing (e.g. source extraction, or overlay with ancillary data).

The common steps involved in the processing of LABOCA data are the following:

- Flux calibration. A correct scaling of the flux involves, at least, two steps: the opacity correction and the counts-to-Jy conversion. The zenith opacity is derived from skydip measurements (see Sects. 8.2.3 and 9.5), and the line of sight opacity also depends on the elevation. The counts-to-Jy factor has been determined during the commissioning of the instrument, but additional correction factors may be applied, depending on the flux measured on calibrators with known fluxes (see Sect. 9.4).

- Flagging of bad channels. Bolometers not responding or with strong excess noise are automatically identified from their rms noise being well outside the main distribution of the rms noise values across the array. They can be flagged, which means that the signal that they recorded is not used any further in the processing.

- Flagging of stationary points. The data acquired when the telescope was too slow to produce a signal inside the useful part of the post-detection frequency band of LABOCA (e.g. below $0.1 \mathrm{~Hz}$, see Sect. 8.1 and Fig. 8) can be flagged. Data obtained when the telescope acceleration is very high may show some excess noise, and can also be flagged.

- Correlated noise removal. This can be done using a Principal Components Analysis (PCA), or a median noise removal method. In the latter case, the median value of all (normalized) signals is computed at each timestamp, and subtracted from the signal of each channel (with appropriate relative gains). This can be performed using all beams at once or better on groups of selected beams. In fact, some groups of channels sharing the same electronics subsystem (e.g. amplifier box, flat cable) can show strong correlation and removing the median signal on those groups of channels greatly improves their signal-to-noise ratio. However, it should be noted that an astronomical source with extended

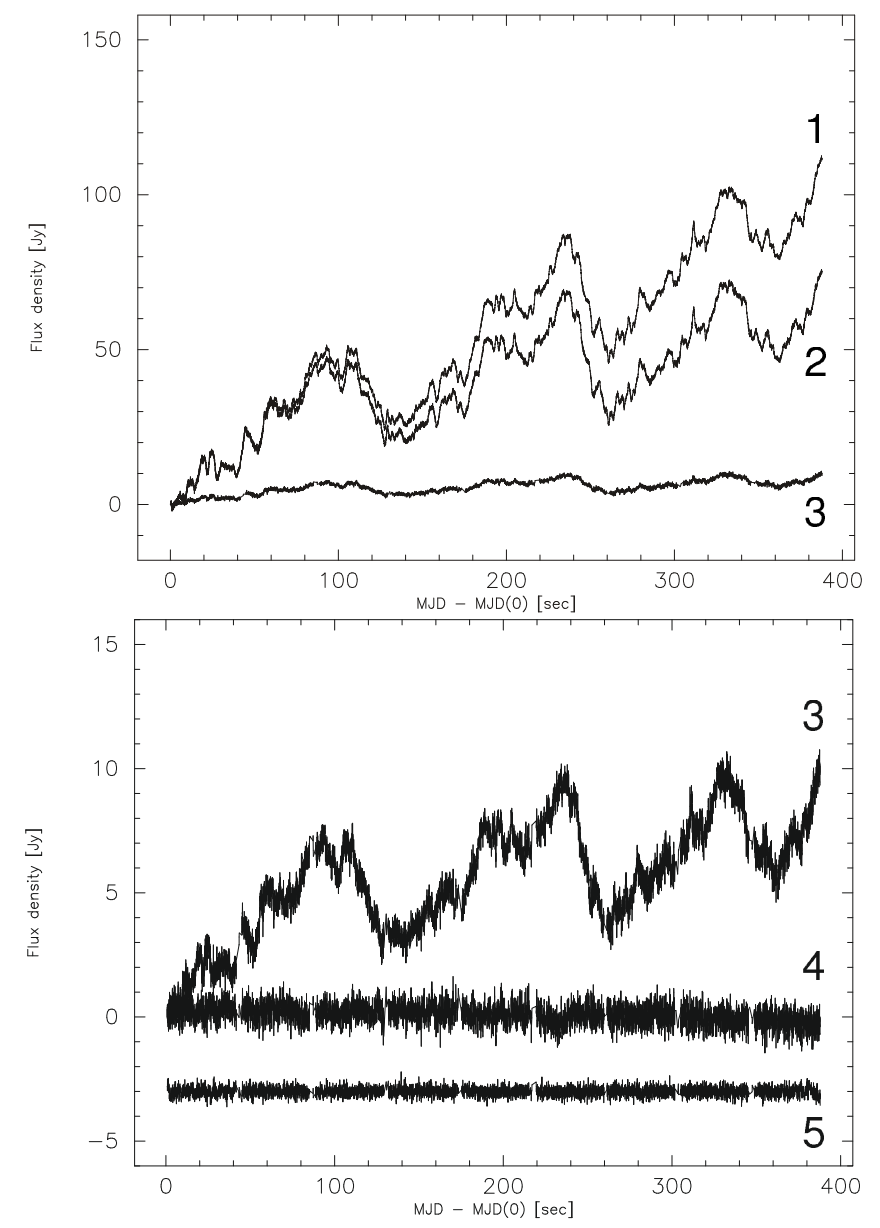

Fig. 14. Time series of a bolometer signal on blank sky at consecutive steps during the data reduction process with BoA. The signals, labeled from 1 to 5 , are shown for: 1) original signal on sky; 2) after correcting for system temperature variations using the blind bolometers ( $200 \mu \mathrm{K}$ during this scan); 3 ) after median skynoise removal over the full array, one single iteration; 4) after median skynoise removal over the full array, 10 iterations; 5) after correlated noise removal, grouping the channels by amplifier and cable, 5 iterations (shifted to a -3 level, for clarity).

uniform emission on scales of several arcmin or larger would mimic the correlated noise behavior of groups of channels. Therefore, subtracting the median noise results in filtering out some fraction of the extended emission, depending on the size and morphology of the source.

- Despiking. Data points that deviate by more than a given factor times the standard deviation in each channel can be flagged.

- Data weighting and map making. To build a map in horizontal or equatorial coordinates, the data of all usable channels are projected onto a regular grid and co-added, using a weighted average, with weights computed as $1 / \mathrm{rms}_{\mathrm{c}}^{2}$, where $\mathrm{rms}_{\mathrm{c}}$ refers to the rms noise of an individual channel. The channel rms noise can either be the standard deviation of the data over the full time line, or it can be computed on a sliding window containing a given number of integrations.

A visual example of correlated noise removal with BoA is given in Fig. 14.

Additional (optional) steps that can improve the final reduction include: removing slow variations from the signal, by subtracting a polynomial baseline or by filtering out low frequencies 
in the Fourier domain; smoothing of the map with a Gaussian kernel of a given size.

The map resulting from a full reduction can be used as a model of the astronomical source to mask the data, in order to repeat some computations without using data points corresponding to the source in the map. This iterative scheme helps in the difficult task to recover the extended emission and reduces the generation of artifacts around strong sources. In the presence of bright sources in the map, a typical data reduction should first perform the reduction steps as listed above with conservative parameters (for example, using high enough thresholds in the despiking to avoid flagging out the strong sources), and should then read again the raw data, use the resulting map to temporarily flag out datapoints corresponding to bright sources, and repeat the full process with less conservative parameters.

In addition to the data processing itself, BoA allows visualization of the data in different ways (signal vs. time, correlation between channels, power spectra or datagrams), as well as the telescope pattern, speed and acceleration. Finally, BoA also includes a simulation module, which can be used to investigate the mapping coverage for on-the-fly maps, spirals and raster of spirals, given the bolometer array parameters.

\section{Science with LABOCA}

Because of its spectral passband, centered at the wavelength of $870 \mu \mathrm{m}$ (see Fig. 5), LABOCA is particularly sensitive to thermal emission from cold objects in the Universe which is of great interest for a number of astrophysical research fields.

\subsection{Planet formation}

The study of Kuiper Belt Objects in the Solar System as well as observations of debris disks of cold dust around nearby main sequence stars can give vital clues to the formation of our own planetary system and planets in general. With its angular resolution of $19^{\prime \prime}$ (see Fig. 11) LABOCA can resolve the debris disks of some nearby stars.

\subsection{Star formation in the Milky Way}

The outstanding power of LABOCA in mapping large areas of sky with high sensitivity (see Fig. 15) makes it possible, for the first time, to perform unbiased surveys of the distribution of the cold dust in the Milky Way. As the dust emission at $870 \mu \mathrm{m}$ is typically optically thin, it is a direct tracer of the gas column density and gas mass. Large scale surveys in the Milky Way will reveal the distribution and gas properties of a large number of pre-star cluster clumps and pre-stellar cores in different environments and evolutionary states. Equally importantly, they provide information on the structure of the interstellar medium on large scales at high spatial resolution, little explored so far. Such surveys are vital to improve our understanding of the processes that govern star formation as well as the relation between the clump mass spectrum and the stellar initial mass function. Large unbiased surveys are also critical for finding precursors of high-mass stars, which are undetectable at other wavelengths due to the high obscuration of the massive cores they are embedded in. LABOCA will help to obtain a detailed understanding of their evolution. In addition, deep surveys of nearby, star-forming clouds, will allow study of the pre-stellar mass function down to the brown dwarf regime.

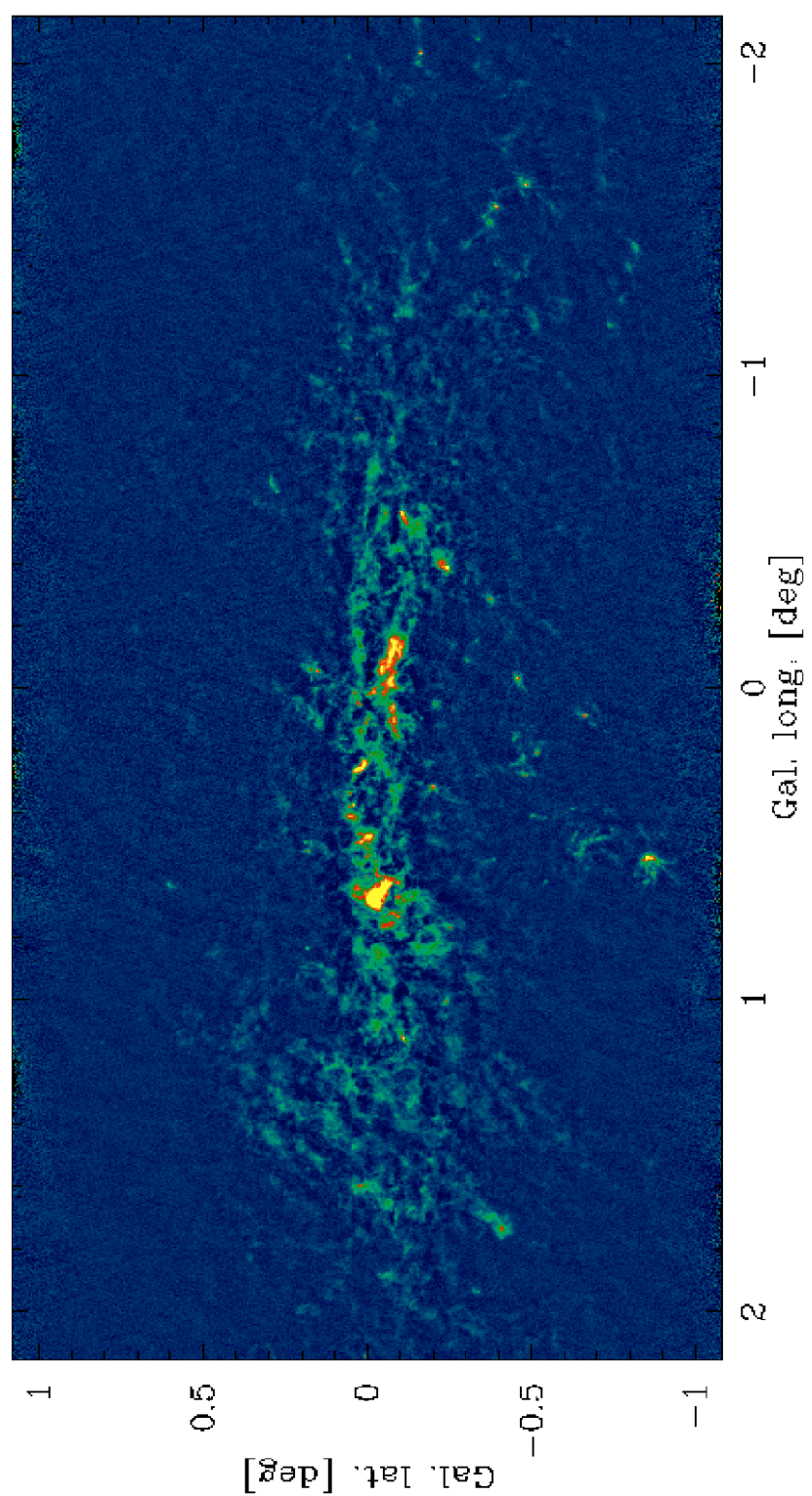

Fig. 15. A map of the galactic center, extracted from the project ATLASGAL (Schuller et al., in prep.). The map is $2 \times 4$ degrees large, the residual noise is $50 \mathrm{mJy}^{-1}$ beam $^{-1}$ and it required only $6 \mathrm{~h}$ of observing time. Data reduced with the BoA package.

\subsection{Cold gas in galaxies}

The only reliable way to trace the bulk of dust in galaxies is through imaging at submillimeter wavelengths. It is becoming clear that most of the dust mass in spiral galaxies lies in cold, low surface-brightness disks, often extending far from the galactic nucleus, as in the case of the starburst galaxy NGC 253 or of NGC 5128 (Cen A, see Fig. 16 and Weiß et al. 2008). Understanding this component is critically important as it dominates the total gas mass in galaxies and studies of the Schmidt law based on H I observations only can heavily underestimate the gas surface density in the outer parts.

In addition to studying individual nearby galaxies, LABOCA will be vital for determining the low- $z$ benchmarks, such as the local luminosity and dust mass functions, which are required to interpret information from deep cosmological surveys. 


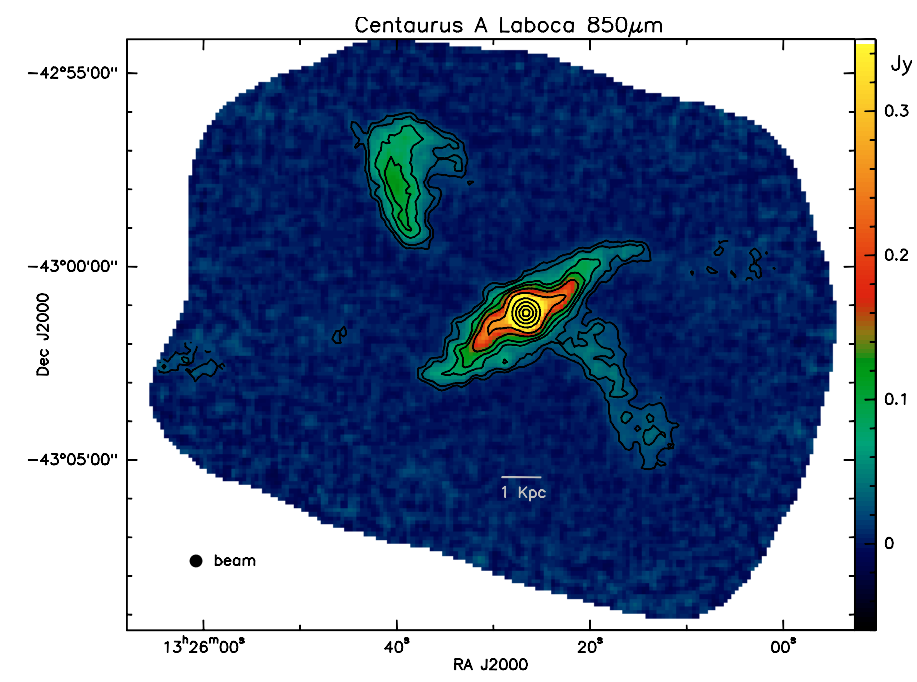

Fig. 16. Map of the nearby galaxy Centaurus A (NGC 5128, from Weiß et al. 2008). The central dust disk has a pronounced S-shape. Our LABOCA image shows, for the first time at submillimeter wavelengths, the synchrotron emission from the radio jets and the inner radio lobes. Its distribution follows closely the known radio emission at $\mathrm{cm}$ wavelengths. The color scale is in units of $\mathrm{Jy} \mathrm{beam}^{-1}$. The residual rms noise is $4 \mathrm{mJy}_{\text {beam }}^{-1}$ and the total integration time is $5 \mathrm{~h}$.

\subsection{Galaxy formation at high redshift}

Due to the negative-K correction, submillimeter observations offer equal sensitivity to dusty star-forming galaxies over a redshift range from $z \sim 1-10$ and therefore provide information on the star formation history at epochs from about half to only $5 \%$ of the present age of the universe. Recent studies have shown that the volume density of luminous submillimeter galaxies (SMGs) increases over a thousand-fold out to $z \sim 2$ (Chapman et al. 2005), and thus, in contrast to the local Universe, luminous obscured galaxies at high redshift could dominate the total bolometric emission from all galaxies at early epochs. The masstracing property of submillimeter dust emission (see Sect. 1.1) allows us to make a direct estimate of the star formation rates (SFRs) of these objects. The generally high observed SFRs suggest that approximately half of all the stars that have formed by the present day may have formed in highly obscured systems which remain undetected at optical or near-infrared wavelengths. One example for such system is the submillimeter galaxy SMM $\mathrm{J} 14009+0252$ (see Fig. 17), which is strong in the submillimeter range, has a $1.4 \mathrm{GHz}$ radio counterpart, but no obvious counterpart in deep $K$-band images (Ivison et al. 2000). Clearly it is critical to include these highly obscured sources in models of galaxy formation to obtain a complete understanding of the evolution of galaxies. With its fast mapping capabilities LABOCA allows us to map fields of a half square degree, a typical size of a deep cosmological field surveyed at other wavelengths, down to the confusion limit in a reasonable amount of observing time. This will also greatly improve the statistics of high redshift galaxies detected at submillimeter wavelengths. See Beelen et al. (2008) for the first deep LABOCA cosmology imaging.

\section{Future plans}

In collaboration with the Institute for Photonics Technology (IPHT, Jena, Germany) we are working on a new bolometer array, LABOCA-2, using superconducting technology. The new

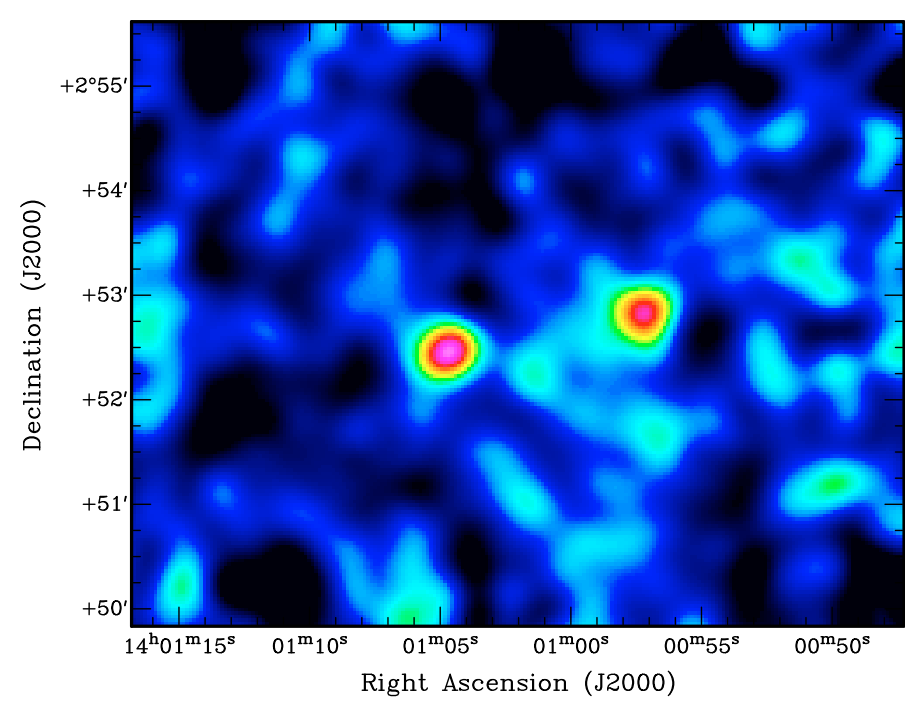

Fig. 17. LABOCA images of SMM J14011+0252 (left) and SMM J14009+0252 (right) smoothed to 25" resolution. SMMJ14011 is at a redshift of $z=2.56$ while SMM J14009 has no reliable redshift determination. The noise level of the map is about $2.5 \mathrm{mJy}$ beam $^{-1}$.

array will use superconducting thermistors (transition edge sensor, TES), planar dipole absorbers, SQUID (Superconducting Quantum Interference Device) multiplexing and amplification. A system already using the same technology, called Submillimeter APEX Bolometer Camera (SABOCA) is going to be commissioned as facility instrument on the APEX telescope for operation in the $350 \mu \mathrm{m}$ atmospheric window. Additionally, given the low sensitivity of superconducting bolometers to microphonics, it will be possible to move from the wet cryostat to a pulse-tube cooler, as already successfully tested at MPIfR in Bonn, improving considerably the conditions of operability of the system.

Acknowledgements. The authors would like to thank the staff at the APEX telescope for their support during installation and commissioning of the instrument. Special thanks to Lars-Åke Nyman (ESO, APEX station manager at the time of the commissioning) for his invaluable help and for his belief in the project even in the most difficult moments. E.K. enjoyed the hospitality and help provided by the staff and many student members of the Microfabrication Facility of UC Berkeley during the manufacture of the LABOCA wafer.

\section{Appendix A: Secondary calibrators}

\section{A.1. The absolute flux scale and secondary calibrators}

The absolute flux density calibration of (sub)millimeter photometry is usually established via observations of planets with well characterized temperature models and angular sizes smaller than the telescope beam (19" for LABOCA on APEX, see 9.3). Thus, the primary calibrators for LABOCA are Mars, Uranus, and Neptune. A planet's flux density is, to first order, determined by its angular size and its temperature at the time of observation, which in turn is determined by its insolation. The situation is complicated, a.o., by diurnal temperature variations and the physics and composition of the planetary atmospheres (see, e.g., Ulich 1981; Hildebrand et al. 1985; Griffin et al. 1986; Orton et al. 1986).

In practice good calibration of science data requires frequent comparisons to calibration sources, preferably near the science 
Table A.1. Properties of the secondary calibrators defined with LABOCA.

\begin{tabular}{|c|c|c|c|c|c|c|c|c|c|}
\hline Source & $\alpha(\mathrm{J} 2000)$ & $\delta(\mathrm{J} 2000)$ & $\begin{array}{c}S_{\mathrm{G}}^{a} \\
\left(\mathrm{Jy} \mathrm{beam}^{-1}\right)\end{array}$ & $\mathrm{Nr}$. & $\begin{array}{c}S_{\text {peak }}^{b} \\
\left(\mathrm{Jy} \mathrm{beam}^{-1}\right)\end{array}$ & $\begin{array}{c}\int S \mathrm{~d} \Omega_{\mathrm{G}}^{c} \\
(\mathrm{Jy})\end{array}$ & $\begin{array}{r}\theta_{\text {maj }} \\
\left({ }^{\prime \prime}\right)\end{array}$ & $\begin{array}{r}\theta_{\min } \\
(")\end{array}$ & $\begin{array}{r}\text { PA } \\
\left({ }^{\circ} \mathrm{E} \text { of } N\right)\end{array}$ \\
\hline CRL 618 & $04^{\mathrm{h}} 42^{\mathrm{m}} 54^{\mathrm{s}} .00$ & $+36^{\circ} 06^{\prime} 53^{\prime \prime} .7$ & $4.9 \pm 0.2$ & 6 & $4.7(2)$ & $5.5(4)$ & $19.7(8)$ & $19.0(8)$ & - \\
\hline V883 ORI & $05^{\mathrm{h}} 38^{\mathrm{m}} 18.10$ & $-07^{\circ} 02^{\prime} 27^{\prime \prime} 0$ & $1.36 \pm 0.11$ & 41 & $1.35(3)$ & $1.93(6)$ & $22.3(5)$ & $21.3(5)$ & $51(20)$ \\
\hline NGC 2071 & $05^{\mathrm{h}} 47^{\mathrm{m}} 04^{\mathrm{s}} .85$ & $+00^{\circ} 21^{\prime} 47 \prime^{\prime \prime} 1$ & $9.1 \pm 0.6$ & 102 & $10.0(1)$ & 26.6(3) & $30.3(3)$ & 28.1(3) & $157(4)$ \\
\hline VY CMa & $07^{\mathrm{h}} 22^{\mathrm{m}} 58.33$ & $-25^{\circ} 46^{\prime} 03^{\prime \prime} .2$ & $1.54 \pm 0.17$ & 34 & $1.52(2)$ & $1.90(5)$ & 21.1(3) & $21.0(3)$ & - \\
\hline IRAS 13134-6264 & $13^{\mathrm{h}} 16^{\mathrm{m}} 43^{\mathrm{s}} .15$ & $-62^{\circ} 58^{\prime} 31^{\prime \prime} 6$ & $12.9 \pm 0.9$ & 134 & $12.9(1)$ & 21.1(2) & $23.5(2)$ & 23.3(2) & - \\
\hline IRAS $16293-2422$ & $16^{\mathrm{h}} 32^{\mathrm{m}} 22^{\mathrm{s}} .90$ & $-24^{\circ} 28^{\prime} 35^{\prime \prime} 6$ & $16.4 \pm 1.2$ & 142 & $17.4(2)$ & $28.5(3)$ & $26.9(2)$ & $24.6(2)$ & $148(3)$ \\
\hline G $10.62-0.38$ & $18^{\mathrm{h}} 10^{\mathrm{m}} 28.66$ & $-19^{\circ} 55^{\prime} 49^{\prime \prime} 7$ & $33.4 \pm 2.0$ & 408 & $35.4(2)$ & $72.0(6)$ & 29.2(2) & $24.5(1)$ & $100(1)$ \\
\hline G $34.26+0.15$ & $18^{\mathrm{h}} 53^{\mathrm{m}} 18.50$ & $+01^{\circ} 14^{\prime} 58^{\prime \prime} 6$ & $55.5 \pm 3.5$ & 164 & $60.1(1)$ & $128.5(6)$ & $27.7(1)$ & $26.2(1)$ & $103(3)$ \\
\hline G $45.07+0.13$ & $19^{\mathrm{h}} 13^{\mathrm{m}} 22^{\mathrm{s}} .07$ & $+10^{\circ} 50^{\prime} 53^{\prime \prime} .^{\prime}$ & $8.2 \pm 0.6$ & 73 & $8.50(5)$ & $15.9(2)$ & $26.2(2)$ & $24.6(2)$ & $67(4)$ \\
\hline $\mathrm{K} 3-50 \mathrm{~A}$ & $20^{\mathrm{h}} 01^{\mathrm{m}} 45.69$ & $+33^{\circ} 32^{\prime} 43^{\prime \prime} .^{\prime}$ & $15.0 \pm 1.2$ & 23 & $16.6(2)$ & 37.7(9) & 31.1(4) & $26.9(3)$ & $49(4)$ \\
\hline CRL 2688 & $21^{\mathrm{h}} 02^{\mathrm{m}} 18.80$ & $+36^{\circ} 41^{\prime} 37^{\prime \prime} 7$ & $5.4 \pm 0.4$ & 20 & $5.3(2)$ & $6.6(1)$ & $23.2(2)$ & $22.5(2)$ & $53(14)$ \\
\hline
\end{tabular}

${ }^{a}$ Mean peak flux derived from a Gaussian fit to all individual scans, errors are the standard deviation of these values.

${ }^{b}$ Peak flux on the co-added map (given in Fig. A.1), errors correspond to the map rms.

${ }^{c}$ Integrated flux of a Gaussian fit on the co-added map, errors are the formal fitting errors.

Columns are (left to right) source name, right ascension and declination (equinox J2000), Gaussian peak flux, number of observations, map peak flux, integrated Gaussian flux, major and the minor axis and mean position angle (east of north). The error for the Gaussian peak flux is the standard deviation of all measurements and gives the typical LABOCA calibration accuracy including systematic effects such as uncertainties in the focus and errors propagated from the opacity determination. The numbers in parentheses are formal fitting uncertainties in the last quoted digit. The overall uncertainties are estimated to be $\sim 10 \%$, including the uncertainties in the observations of the primary calibrators (Mars, Uranus, Neptune).

target, to eliminate systematic calibration effects (mostly elevation dependent). For this reason, the small number of primary calibrators (only three for LABOCA), cannot serve all scientific calibration needs. It is therefore highly desirable to have a list of additional compact objects that serve as secondary calibrators provided their fluxes have been determined with particular care via comparisons to the primary calibration sources. A variety of secondary calibrators, with flux densities determined at various wavelengths $350 \mu \mathrm{m}<\lambda<2 \mathrm{~mm}$, using the $15 \mathrm{~m}$ James Clerk Maxwell Telescope (JCMT), has been published by Sandell (1994).

\section{A.2. LABOCA observations of secondary calibrators}

Since its commissioning, in May 2007, we have used LABOCA at APEX to observe a number of sources which serve as secondary calibrators. The sample, which is partially based on Sandell (1994), consists of ultracompact HII regions, protostellar objects, and AGB stars. LABOCA observations of secondary calibrators are typically done in raster-spiral mode leading to a fully sampled map for each scan. In Fig. A.1 we present images centered on the LABOCA secondary calibrators, which have been calculated by averaging several raster-spiral scans for each source. In Table A.1 we summarize their properties.

For each scan, we fitted elliptical Gaussian profiles to the observed flux density distribution. The flux values quoted in the table are averages, while the uncertainties represent the rms scatter of the individually fitted peaks. The fitting errors are generally much smaller than the intrinsic calibration scatter. The quoted uncertainties, therefore, represent the typical LABOCA calibration accuracy including systematic effects such as uncertainties in the focus and errors propagated from the opacity determination.

The typical uncertainty of the secondary calibrator fluxes is about $7 \%$, comparable to the $8 \%$ scatter on the primary calibrators (namely Mars, Uranus and Neptune, 700 observations) derived in the same way and using the flux predictions from the ASTRO model (see Sect. 9.4). We are thus confident that the calibrator fluxes listed in Table A.1 are accurate to $\sim 10 \%$.

In addition to the averaged Gaussian peak flux densities (brightness) we also list, for each calibrator source, the peak flux density measured on the co-added maps (which differs from the Gaussian peak value for extended, structured sources), the flux integrated over a Gaussian fitted to the images shown in Fig. A.1, the apparent major and minor axes and the mean position angles.

\section{A.3. Discussion}

Most of the secondary calibrators defined with LABOCA are also included in the study of Sandell (1994), where photometric flux densities in the JCMT beam are quoted. At the wavelength $\lambda=850 \mu \mathrm{m}$, the closest to the LABOCA's center wavelength among the ones used by Sandell, the reported beam is $17 . " 5$, comparable to LABOCA's 19" (see Sect. 4.1).

Comparison of the peak flux densities measured with LABOCA and the ones from Sandell (1994) shows remarkable agreement. Our peak flux densities on the maps (which is the appropriate value for comparison to the photometric flux, in particular, for non-Gaussian, extended sources) agree with photometric values given by Sandell to within 5\% on average (excluding VY CMa). VY CMa is, in fact, known to be variable (Knapp \& Woodhams 1993) and our 2007-2008 measurements show flux densities about 40\% lower than the values observed in 19891991 by Sandell.

The secondary calibrators all have some line emission (e.g. $\mathrm{CO}(3-2)$ ), which might be a significant fraction of the total for some of them. The line contamination in the fluxes measured by LABOCA, however, has no impact on the calibration of LABOCA science data. 

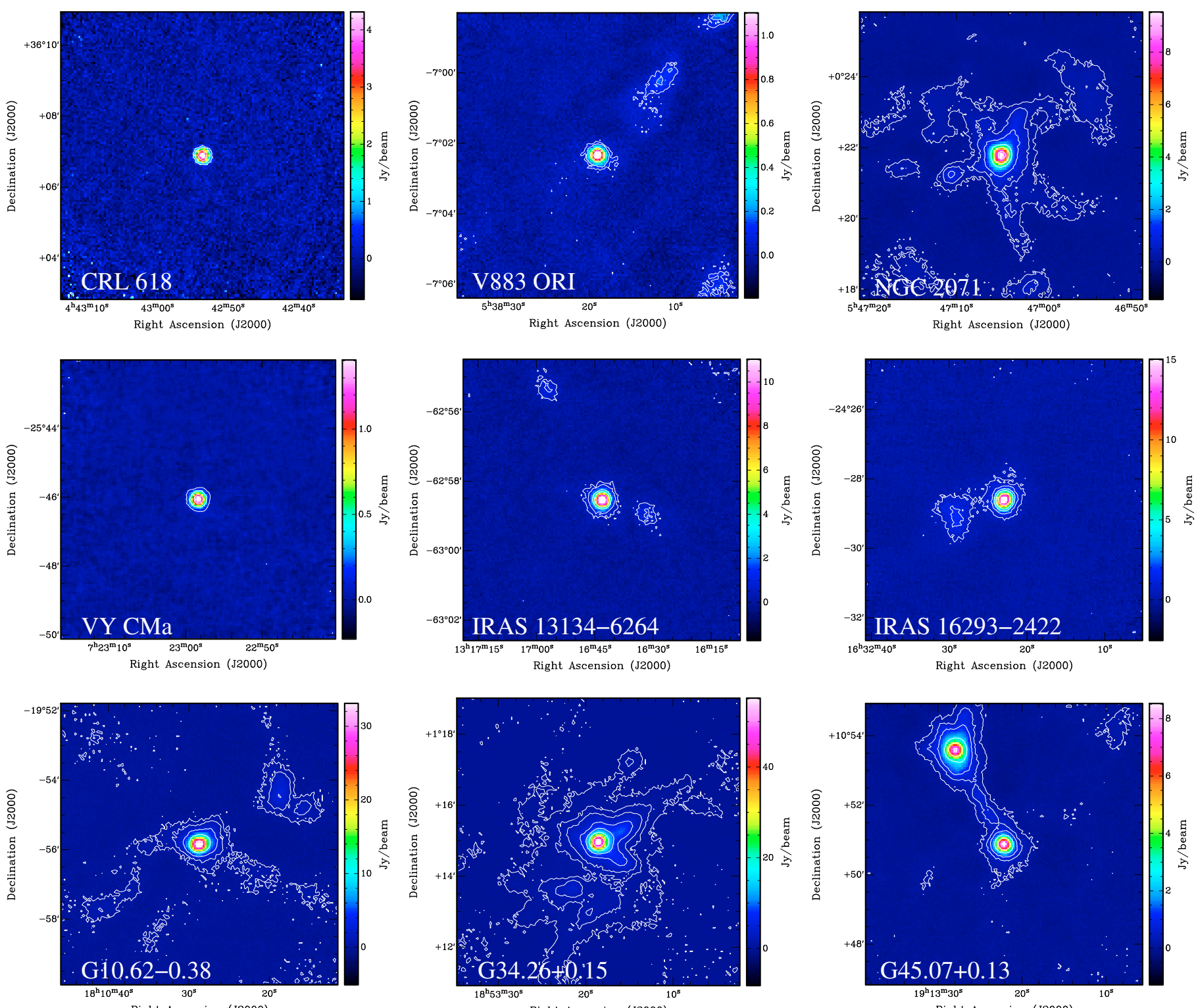

Right Ascension (J2000)

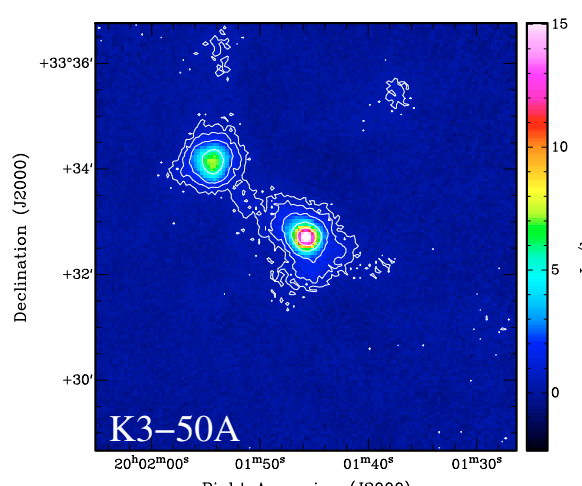

Right Ascension (J2000)

Right Ascension (J2000)

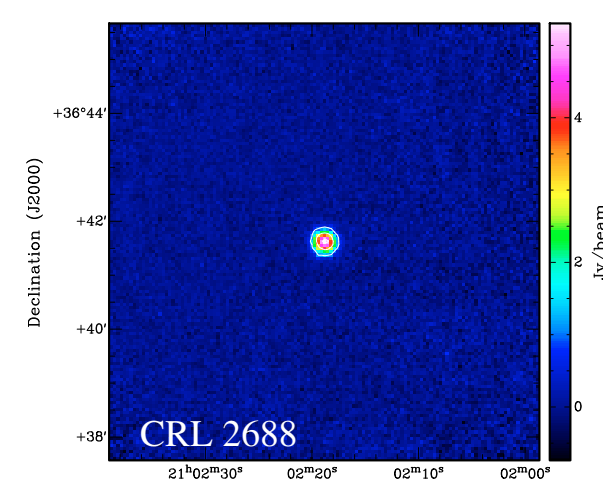

Fig. A.1. Maps of the secondary calibrators imaged with LABOCA (ordered in right ascension). Contours are shown at: $0.75,1.5,3.0 \mathrm{Jy}$ beam ${ }^{-1}$ for CRL 618; 0.08, 0.15, 0.5, $1.0 \mathrm{Jy}_{\text {beam }}^{-1}$ for V883 ORI; 0.1, 0.4, 1.0, 3.0, 5.0, 8.5 Jy beam ${ }^{-1}$ for NGC 2071; 0.1, 0.4, 1.0, $1.4 \mathrm{Jy}_{\text {beam }}{ }^{-1}$ for VY CMa; 0.5, 1.0, 3.0, 5.0, $11.0 \mathrm{Jy}_{\text {beam }^{-1}}$ for IRAS 13134-6264; 0.6, 1.5, 3.0, 5.0, 10.0, $15.0 \mathrm{Jy}^{-1}$ beam $^{-1}$ for IRAS 16293-2422; 0.3, 1.0 3.0 10.0, $30.0 \mathrm{Jy}_{\text {beam }^{-1}}$ for G10.62-0.38; 0.2, 1.0, 2.0, 5.0, 10.0, 20.0, 40.0, 55.0 Jy beam ${ }^{-1}$ for G34.26+0.15; 0.13, 0.5, 1.0 2.5, 5.0, 8.0 Jy beam ${ }^{-1}$ for $\mathrm{G} 45.12+0.13 ; 0.5,1.0,2.0,5.0,10.0,15.0 \mathrm{Jy} \mathrm{beam}^{-1}$ for K3-50A; $1.03 .0,5.0 \mathrm{Jy} \mathrm{beam}^{-1}$ for CRL 2688. 


\section{References}

Beelen, A., Omont, A., Bavouzet, N., et al. 2008, A\&A, 485, 645 Bertoldi, F., Carilli, C. L., Cox, P., et al. 2003, A\&A, 406, L55 Beuther, H., Schilke, P., Menten, K. M., et al. 2002, ApJ, 566, 945 Blain, A. W., \& Longair, M. S. 1993, MNRAS, 264, 509

Blain, A. W., Smail, I., Ivison, R. J., Kneib, J.-P., \& Frayer, D. T. 2002, Phys. Rep., 369, 111

Carilli, C. L., Bertoldi, F., Rupen, M. P., et al. 2001, ApJ, 555, 625

Chapman, I. M., Naylor, D. A., \& Phillips, R. R. 2004, MNRAS, 354, 621

Chapman, S. C., Blain, A. W., Smail, I., \& Ivison, R. J. 2005, ApJ, 622, 772

Duband, L., Clerc, L., \& Ravex, A. 2002, in Advances in Cryogenic Engineering CEC, ed. S. Breon, M. Dipirro, D. Glaister, J. Hull, P. Kittel, V. R. R. Pecharsky, J. Theilacker, S. van Sciver, J. I. Weisend, \& A. Zeller, Amer. Inst. Phys. Conf. Ser., 613, 1233

Griffin, M. J., Ade, P. A. R., Orton, G. S., et al. 1986, Icarus, 65, 244

Güsten, R., Nyman, L. Å., Schilke, P., et al. 2006, A\&A, 454, L13

Haller, E. E., Palaio, N. P., Rodder, M., Hansen, W. L., \& Kreysa, E. 1982, Presented at the 4th Intern. Neutron Transmutation Doping Conf., Gaithersburg, Md., 1-3 June, 83, 28412

Hildebrand, R. H., Loewenstein, R. F., Harper, D. A., et al. 1985, Icarus, 64, 64 Holland, W. S., Robson, E. I., Gear, W. K., et al. 1999, MNRAS, 303, 659

Ivison, R. J., Smail, I., Barger, A. J., et al. 2000, MNRAS, 315, 209

Johnstone, D., Matthews, H., \& Mitchell, G. F. 2006, ApJ, 639, 259

Knapp, G. R., \& Woodhams, M. 1993, in Massive Stars: Their Lives in the Interstellar Medium, ed. J. P. Cassinelli, \& E. B. Churchwell, ASP Conf. Ser., 35,199
Kovács, A. 2008, in SPIE Conf. Ser., 7020

Kreysa, E. 1990, in Liege International Astrophysical Colloquia, ed. B. Kaldeich, Liege International Astrophysical Colloquia, 29, 265

Kreysa, E., Gemünd, H.-P., Gromke, J., et al. 1999, Infrared Physics and Technology, 40, 191

Kreysa, E., Gemünd, H.-P., Raccanelli, A., Reichertz, L. A., \& Siringo, G. 2002, in Experimental Cosmology at Millimetre Wavelengths, ed. M. de Petris, \& M. Gervasi, American Institute of Physics Conf. Ser., 616, 262

Low, F. J. 1961, J. Optical Society of America (1917-1983), 51, 1300

Martin, R. N., \& Baars, J. W. M. 1990, in SPIE Conf. Ser., 1235, 503 Mather, J. C. 1984, Appl. Opt., 23, 584

Mezger, P. G., Zylka, R., \& Wink, J. E. 1990, A\&A, 228, 95

Motte, F., Andre, P., \& Neri, R. 1998, A\&A, 336, 150

Motte, F., Bontemps, S., Schilke, P., et al. 2007, A\&A, 476, 1243

Muders, D., Hafok, H., Wyrowski, F., et al. 2006, A\&A, 454, L25

Nyman, L.-A., Lerner, M., Nielbock, M., et al. 2001, The Messenger, 106, 40

Orton, G. S., Griffin, M. J., Ade, P. A. R., Nolt, I. G., \& Radostitz, J. V. 1986, Icarus, 67, 289

Pardo, J. R., Cernicharo, J., \& Serabyn, E. 2001, IEEE Trans. Antennas Propag., 49, 1683

Reichertz, L. A., Weferling, B., Esch, W., \& Kreysa, E. 2001, A\&A, 379, 735

Sandell, G. 1994, MNRAS, 271, 75

Schwan, D., Bertoldi, F., Cho, S., et al. 2003, New Astron. Rev., 47, 933

Siringo, G., Kreysa, E., Reichertz, L. A., \& Menten, K. M. 2004, A\&A, 422, 751

Ulich, B. L. 1981, AJ, 86, 1619

Wang, R., Wagg, J., Carilli, C. L., et al. 2008, AJ, 135, 1201

Weiß, A., Kovács, A., Güsten, R., et al. 2008, A\&A, 490, 77 